\title{
FREDHOLM DETERMINANTS AND THE EVANS FUNCTION FOR DIFFERENCE EQUATIONS
}

\author{
DAVID CRAMER and YURI LATUSHKIN \\ Department of Mathematics, University of Missouri, Columbia, MO 65211, U.S.A. \\ E-mail: mathgr6@math.missouri.edu,yuri@math.missouri.edu
}

\begin{abstract}
We develop a difference equations analogue of recent results by F. Gesztesy, K. A. Makarov, and the second author relating the Evans function and Fredholm determinants of operators with semi-separable kernels.
\end{abstract}

1. Introduction. The purpose of this paper is to provide a difference equations version of some of the most recent results in [GM, GML, GML1] relating the Evans function and Fredholm determinants of operators with semi-separable kernels. Although our general strategy is close to that in [GM, GML] for the differential equations case, and for simplicity we consider less general assumptions than in [GML], the arguments and the results in the difference equations setting have some important differences. For a related work we cite [GGK, GKvS] and [BCK, KK]. For a detailed historical account and the bibliography we refer to [GML].

We consider an unperturbed difference equation $x_{j+1}=A_{j} x_{j}$ and its perturbation in the form $x_{j+1}=A_{j}^{\times} x_{j}, j \in \mathbb{Z}$, where $A_{j}^{\times}=A_{j}+B_{j} C_{j}$. Here and below, $A_{j}, B_{j}$, and $C_{j}$ are $(\mathrm{d} \times \mathrm{d})$ matrices with complex entries, and $\mathbf{x}=\left(x_{j}\right)_{j \in \mathbb{Z}}$ is a sequence of vectors $x_{j} \in \mathbb{C}^{\mathrm{d}}$. Throughout, we assume that the matrices $A_{j}$ and $A_{j}^{\times}$are invertible and that the unperturbed equation has an exponential dichotomy over $\mathbb{Z}$ with the (unstable) dichotomy projection $P$. Let $\mathbf{U}=\left(U_{j}\right)_{j \in \mathbb{Z}}$ denote the fundamental matrix solution of the unperturbed equation normalized by $U_{0}=I$, the identity matrix.

2000 Mathematics Subject Classification: Primary 34D09, 47B39; Secondary 39xx, 34D08.

Key words and phrases: Fredholm determinants, Evans function, Lyapunov exponents.

Research of the second author was supported in part by the NSF grants 0338743 and 0354339 , by the CRDF grant UP1-2567-OD-03 and by the Research Board and the Research Council of the University of Missouri.

The authors thank F. Gesztesy for helpful discussions and K. A. Makarov for many valuable suggestions.

The paper is in final form and no version of it will be published elsewhere. 
In the first part of the paper, following [GM], we give formulas for the (modified) Fredholm determinants of the difference operator $\mathcal{T}=\left(T_{j k}\right)_{j, k \in \mathbb{Z}}$ on $\ell^{2}\left(\mathbb{Z} ; \mathbb{C}^{\mathrm{d}}\right)$ whose kernel is given by the formulas

$$
T_{j k}=C_{j} U_{j}(I-P) U_{k+1}^{-1} B_{k} \text { for } j>k \text { and } T_{j k}=-C_{j} U_{j} P U_{k+1}^{-1} B_{k} \text { for } j \leq k .
$$

Note that the kernel of every difference operator with a semi-separable kernel admits a representation (1.1), see formulas (3.9) - (3.10) below. The choice of kernel (1.1) is related to the following elementary observation. Given the matrix sequence $\mathbf{A}=\left(A_{j}\right)_{j \in \mathbb{Z}} \in$ $\ell^{\infty}\left(\mathbb{Z} ; \mathbb{C}^{\mathrm{d} \times \mathrm{d}}\right)$, define on $\ell^{2}\left(\mathbb{Z} ; \mathbb{C}^{\mathrm{d}}\right)$ an operator, $G_{\mathbf{A}}$, by $\left(G_{\mathbf{A}} \mathbf{x}\right)_{j}=x_{j+1}-A_{j} x_{j}$ so that the inhomogeneous equations $x_{j+1}=A_{j} x_{j}+y_{j}, j \in \mathbb{Z}$, becomes $G_{\mathbf{A}} \mathbf{x}=\mathbf{y}$. Due to the exponential dichotomy [CL], the operator $G_{\mathbf{A}}$ is invertible in $\ell^{2}\left(\mathbb{Z}, \mathbb{C}^{\mathrm{d}}\right)$, and by a direct computation, its inverse is a difference operator, $\mathcal{K}=\left(K_{j k}\right)_{j, k \in \mathbb{Z}}$, with kernel defined by

$$
K_{j k}=U_{j}(I-P) U_{k+1}^{-1} \text { for } j>k \text { and } K_{j k}=-U_{j} P U_{k+1}^{-1} \text { for } j \leq k .
$$

If $\mathbf{A}^{\times}=\left(A_{j}^{\times}\right)_{j \in \mathbb{Z}}$ then the operator $G_{\mathbf{A}^{\times}}=G_{\mathbf{A}}-\operatorname{diag}\left(B_{j} C_{j}\right)_{j \in \mathbb{Z}}$ can be represented as $G_{\mathbf{A}^{\times}}=G_{\mathbf{A}}\left(I-\mathcal{K} \operatorname{diag}\left(B_{j}\right)_{j \in \mathbb{Z}} \operatorname{diag}\left(C_{j}\right)_{j \in \mathbb{Z}}\right)$, and $G_{\mathbf{A} \times}$ is invertible if and only if the operator $I-\mathcal{T}$ is invertible; the kernel of $\mathcal{T}=\operatorname{diag}\left(C_{j}\right)_{j \in \mathbb{Z}} \mathcal{K} \operatorname{diag}\left(B_{j}\right)_{j \in \mathbb{Z}}$ is given by (1.1).

In the second part of the paper, following [GML, GML1], we construct appropriate matrix solutions of the perturbed difference equation whose determinant, $\mathcal{E}$, is called the Evans determinant. If the sequence $\mathbf{A}^{\times}=\mathbf{A}^{\times}(z)$ depends on a spectral parameter $z \in \mathbb{C}$, then the corresponding function $\mathcal{E}=\mathcal{E}(z)$ becomes the Evans function, a Wronskian type object widely used to detect unstable modes for operators obtained by linearizing nonlinear equations along special particular solutions such as travelling waves, see [AGJ] and recent reviews $[\mathrm{JK}, \mathrm{S}]$ and the bibliographies therein. We stress that the Evans determinant, as defined in the current paper, is uniquely determined by the sequences $\mathbf{A}^{\times}$and $\mathbf{A}$. Moreover (and this is the central result of this paper), we derive a formula relating $\mathcal{E}$ and the Fredholm determinant of $I-\mathcal{T}$ (for results in this spirit in the case of the Schrödinger differential operator see [KS, p. 861] and [KS1]). Finally, for the discrete Schrödinger operator, we show that the Evans function coincides with the Jost function, the classical object familiar from scattering theory, see e.g. [CS, Chap. XVII], [FT, Sec. III.2], [GH, Sec. 6], [T, Chap. 10], and [To, Chap. 3].

2. Notation and preliminaries. The set of $(d \times d)$ matrices with complex entries is denoted by $\mathbb{C}^{\mathrm{d} \times \mathrm{d}}$. Where possible, we abbreviate $\ell^{2}=\ell^{2}\left(\mathbb{Z} ; \mathbb{C}^{\mathrm{d}}\right)$ or $\ell^{2}=\ell^{2}\left(\mathbb{Z} ; \mathbb{C}^{\mathrm{d} \times \mathrm{d}}\right)$. We use boldface to denote sequences of vectors or matrices, e.g. $\mathbf{x}=\left(x_{j}\right)_{j \in \mathbb{Z}}, x_{j} \in \mathbb{C}^{\mathrm{d}}$, or $\mathbf{a}=\left(a_{j}\right)_{j \in \mathbb{Z}}, a_{j} \in \mathbb{C}^{\mathrm{d} \times \mathrm{d}}$. We denote by $\sigma(\cdot)$ the spectrum of an operator, and by $I$ (or sometimes $\left.I_{\mathrm{d} \times \mathrm{d}}\right)$ the identity operator. For a projection $P$ on $\mathbb{C}^{\mathrm{d}}$ with $\operatorname{dim} \operatorname{Im} P=\mathrm{d}_{1}$ we often identify $I_{\mathrm{d}_{1} \times \mathrm{d}_{1}}$ and $P$ on $\operatorname{Im} P$. The restriction of an operator $A$ on a subspace $(\cdot)$ is denoted by $\left.A\right|_{(\cdot)}$. If $A$ satisfies $A=A P$ then we denote $\|A\|_{\bullet}=\inf \{\|A x\|: x=$ $P x,\|x\|=1\}$.

The sets of trace-class and Hilbert-Schmidt operators on a Hilbert space $(\cdot)$ are denoted, respectively, by $\mathcal{B}_{1}=\mathcal{B}_{1}(\cdot)$ and $\mathcal{B}_{2}=\mathcal{B}_{2}(\cdot)$. Recall that $\ell^{1} \subset \ell^{2} \subset \ell^{\infty}$ and $\mathcal{B}_{1}(\cdot) \subset \mathcal{B}_{2}(\cdot)$. We will use the following properties of the (modified) Fredholm determinants, see, e.g. [GGK, Si] for more information: 


$$
\begin{aligned}
\operatorname{det}(I-A) & =\prod_{\lambda \in \sigma(A)}(1-\lambda), \quad A \in \mathcal{B}_{1}, \\
\operatorname{det}_{2}(I-A) & =\operatorname{det}\left[(I-A) e^{A}\right]=\prod_{\lambda \in \sigma(A)}(1-\lambda) e^{\lambda}, \quad A \in \mathcal{B}_{2}, \\
\operatorname{det}_{2}(I-A) & =\operatorname{det}(I-A) e^{\operatorname{tr} A}, \quad A \in \mathcal{B}_{1}, \\
\operatorname{det}_{2}[(I-A)(I-B)] & =\operatorname{det}_{2}(I-A) \operatorname{det}_{2}(I-B) e^{-\operatorname{tr}(A B)}, \quad A, B \in \mathcal{B}_{2} .
\end{aligned}
$$

Given matrix sequences $\mathbf{a}_{j}=\left(a_{j}\right)_{j \in \mathbb{Z}}, \mathbf{b}_{j}=\left(b_{j}\right)_{j \in \mathbb{Z}}$, and $\mathbf{d}_{j}=\left(d_{j}\right)_{j \in \mathbb{Z}}$, we define the upper triangular operator $V_{\mathbf{a}, \mathbf{b}}^{+}$, the lower triangular operator $V_{\mathbf{a}, \mathbf{b}}^{-}$, the diagonal operator $D_{\mathbf{d}}$, and the operator $V_{\mathbf{a}, \mathbf{b}}$ on $\ell^{2}\left(\mathbb{Z} ; \mathbb{C}^{\mathrm{d}}\right)$ as follows:

$$
\begin{aligned}
\left(V_{\mathbf{a}, \mathbf{b}}^{+} \mathbf{x}\right)_{j} & =\sum_{k=j+1}^{\infty} a_{j} b_{k} x_{k}, \quad\left(V_{\mathbf{a}, \mathbf{b}}^{-} \mathbf{x}\right)_{j}=\sum_{k=-\infty}^{j-1} a_{j} b_{k} x_{k}, \\
\left(D_{\mathbf{d}} \mathbf{x}\right)_{j} & =d_{j} x_{j}, \quad\left(V_{\mathbf{a}, \mathbf{b}} \mathbf{x}\right)_{j}=\sum_{k=-\infty}^{\infty} a_{j} b_{k} x_{k}, \quad j \in \mathbb{Z} .
\end{aligned}
$$

We summarize properties of these operators in the following elementary lemmas.

Lemma 2.1. Assume $\mathbf{a}, \mathbf{b}, \mathbf{d} \in \ell^{2}\left(\mathbb{Z} ; \mathbb{C}^{\mathrm{d} \times \mathrm{d}}\right)$. Then:

$$
\begin{aligned}
& \sigma\left(D_{\mathbf{d}}\right)=\{0\} \cup\left(\cup_{j \in \mathbb{Z}} \sigma\left(d_{j}\right)\right) \\
& V_{\mathbf{a}, \mathbf{b}}, V_{\mathbf{a}, \mathbf{b}}^{ \pm}, D_{\mathbf{d}} \in \mathcal{B}_{2}\left(\ell^{2}\left(\mathbb{Z} ; \mathbb{C}^{\mathrm{d}}\right)\right) ; \\
& \sigma\left(V_{\mathbf{a}, \mathbf{b}}^{+}\right)=\sigma\left(V_{\mathbf{a}, \mathbf{b}}^{-}\right)=\{0\} \\
& \operatorname{det}_{2}\left(I-V_{\mathbf{a}, \mathbf{b}}^{+}\right)=\operatorname{det}_{2}\left(I-V_{\mathbf{a}, \mathbf{b}}^{-}\right)=1 ; \\
& \operatorname{det}_{2}\left(I-D_{\mathbf{d}}\right)=\prod_{j \in \mathbb{Z}} \operatorname{det}\left(I_{\mathrm{d} \times \mathrm{d}}-d_{j}\right) e^{\operatorname{tr} d_{j}} .
\end{aligned}
$$

Lemma 2.2. Assume $\mathbf{a}, \mathbf{b}, \mathbf{d} \in \ell^{1}\left(\mathbb{Z} ; \mathbb{C}^{\mathrm{d} \times \mathrm{d}}\right)$. Then:

$V_{\mathbf{a}, \mathbf{b}}^{ \pm}$are compact operators on $\ell^{\infty}\left(\mathbb{Z} ; \mathbb{C}^{\mathrm{d}}\right)$ and $\sigma\left(V_{\mathbf{a}, \mathbf{b}}^{ \pm}\right)=\{0\} ;$

$$
\begin{aligned}
& V_{\mathbf{a}, \mathbf{b}}, V_{\mathbf{a}, \mathbf{b}}^{ \pm}, D_{\mathbf{d}} \in \mathcal{B}_{1}\left(\ell^{2}\left(\mathbb{Z} ; \mathbb{C}^{\mathrm{d}}\right)\right) ; \\
& \operatorname{det}\left(I-V_{\mathbf{a}, \mathbf{b}}^{+}\right)=\operatorname{det}\left(I-V_{\mathbf{a}, \mathbf{b}}^{-}\right)=1 ; \\
& \operatorname{det}\left(I-D_{\mathbf{d}}\right)=\prod_{j \in \mathbb{Z}} \operatorname{det}\left(I_{\mathrm{d} \times \mathrm{d}}-d_{j}\right) .
\end{aligned}
$$

Proof. If $\mathbf{d} \in \ell^{2}$ then $\left\|d_{j}\right\| \rightarrow 0$ as $|j| \rightarrow \infty$ and then, for any $r>0$, there are only finitely many $d_{j}$ 's having $\lambda \in \sigma\left(d_{j}\right)$ with $|\lambda| \geq r$. The formula $\sigma\left(D_{\mathbf{d}}\right)=\operatorname{closure}\left(\cup_{j \in \mathbb{Z}} \sigma\left(d_{j}\right)\right)$ now implies (2.7).

Note that $V_{\mathbf{a}, \mathbf{b}}=D_{\mathbf{d}}+V_{\mathbf{a}, \mathbf{b}}^{+}+V_{\mathbf{a}, \mathbf{b}}^{-}$with $\mathbf{d}=\left(a_{j} b_{j}\right)_{j \in \mathbb{Z}}$ and $V_{\mathbf{a}, \mathbf{b}}^{-}=\left(V_{\mathbf{b}^{*}, \mathbf{a}^{*}}^{+}\right)^{*}$ with

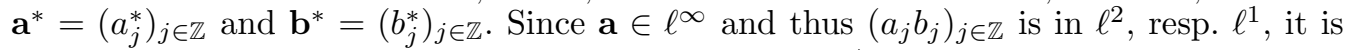
enough to prove (2.9) and (2.8), resp. (2.13), only for $V_{\mathbf{a}, \mathbf{b}}^{+}$and $D_{\mathbf{d}}$. Using (2.7), we infer:

$$
\begin{gathered}
\left\|D_{\mathbf{d}}\right\|_{\mathcal{B}_{1}\left(\ell^{2}\right)}=\operatorname{tr}\left[\left(D_{\mathbf{d}}^{*} D_{\mathbf{d}}\right)^{\frac{1}{2}}\right]=\operatorname{tr}\left[D_{\left(\mathbf{d}^{*} \mathbf{d}\right)^{\frac{1}{2}}}\right]=\sum_{j \in \mathbb{Z}} \operatorname{tr}\left(d_{j}^{*} d_{j}\right)^{\frac{1}{2}} \leq \mathrm{d} \sum_{j \in \mathbb{Z}}\left\|d_{j}\right\|=\mathrm{d}\|\mathbf{d}\|_{\ell^{1}}, \\
\left\|D_{\mathbf{d}}\right\|_{\mathcal{B}_{2}\left(\ell^{2}\right)}=\operatorname{tr}\left(D_{\mathbf{d}^{*} \mathbf{d}}\right)=\sum_{j \in \mathbb{Z}} \operatorname{tr}\left(d_{j}^{*} d_{j}\right) \leq \mathrm{d} \sum_{j \in \mathbb{Z}}\left\|d_{j}\right\|^{2}=\mathrm{d}\|\mathbf{d}\|_{\ell^{2}} .
\end{gathered}
$$


Assuming $\mathbf{a}, \mathbf{b} \in \ell^{1}$ and writing $V_{\mathbf{a}, \mathbf{b}}^{+}=\sum_{k=1}^{\infty} \mathcal{S}^{-k} D_{\left(a_{j-k} b_{j}\right)_{j \in \mathbb{Z}}}$, where $(\mathcal{S} \mathbf{x})_{j}=x_{j-1}$ is the shift operator, we have:

$$
\begin{aligned}
\left\|V_{\mathbf{a}, \mathbf{b}}^{+}\right\|_{\mathcal{B}_{1}\left(\ell^{2}\right)} & \leq \sum_{k=1}^{\infty}\left\|D_{\left(a_{j-k} b_{j}\right)_{j \in \mathbb{Z}}}\right\|_{\mathcal{B}_{1}\left(\ell^{2}\right)}=\sum_{k=1}^{\infty} \sum_{j \in \mathbb{Z}}\left\|a_{j-k} b_{j}\right\|_{\mathcal{B}_{1}\left(\mathbb{C}^{\mathrm{d}}\right)} \\
& \leq \mathrm{d} \sum_{j \in \mathbb{Z}} \sum_{k=1}^{\infty}\left\|b_{j}\right\|\left\|a_{j-k}\right\| \leq \mathrm{d}\|\mathbf{a}\|_{\ell^{1}}\|\mathbf{b}\|_{\ell^{1}} .
\end{aligned}
$$

Assuming $\mathbf{a}, \mathbf{b} \in \ell^{2}$ and considering the basis $\mathbf{y}_{n, i}=\left(y_{n, i}(j)\right)_{j \in \mathbb{Z}}, i=1, \ldots, \mathrm{d}, n \in \mathbb{Z}$, in $\ell^{2}\left(\mathbb{Z} ; \mathbb{C}^{\mathrm{d}}\right)$ given by $y_{n, i}(j)=0$ for $j \neq n$ and $y_{n, i}(n)=\mathbf{e}_{i}$, the standard ort in $\mathbb{C}^{\mathrm{d}}$, we have:

$$
\begin{aligned}
\left\|V_{\mathbf{a}, \mathbf{b}}^{+}\right\|_{\mathcal{B}_{2}\left(\ell^{2}\right)}^{2} & =\operatorname{tr}\left[\left(V_{\mathbf{a}, \mathbf{b}}^{+}\right)^{*} V_{\mathbf{a}, \mathbf{b}}^{+}\right]=\sum_{n \in \mathbb{Z}} \sum_{i=1}^{\mathrm{d}}\left\|V_{\mathbf{a}, \mathbf{b}}^{+} \mathbf{y}_{n, i}\right\|_{\ell^{2}}^{2} \\
& =\sum_{n \in \mathbb{Z}} \sum_{i=1}^{\mathrm{d}} \sum_{j \in \mathbb{Z}}\left\|a_{j} \sum_{k=j+1}^{\infty} b_{k} y_{n, i}(k)\right\|^{2}=\sum_{n \in \mathbb{Z}} \sum_{i=1}^{\mathrm{d}} \sum_{j=n-1}^{\infty}\left\|a_{j} b_{n} \mathrm{e}_{i}\right\|^{2} \\
& \leq \mathrm{d}\|\mathbf{a}\|_{\ell^{2}}^{2}\|\mathbf{b}\|_{\ell^{2}}^{2} .
\end{aligned}
$$

This proves (2.8) and (2.13). To prove (2.9), observe that $\sigma\left(V_{\mathbf{a}, \mathbf{b}}^{+}\right)$consists of eigenvalues since the operator $V_{\mathbf{a}, \mathbf{b}}^{+}$is compact by (2.8). Suppose there are $\lambda \neq 0$ and $\mathbf{0} \neq \mathbf{x} \in$ $\ell^{2}\left(\mathbb{Z} ; \mathbb{C}^{\mathrm{d}}\right)$, so that $a_{j} \sum_{k=j+1}^{\infty} b_{k} x_{k}=\lambda x_{j}$. Then

$$
\sum_{j=n+1}^{\infty} c_{j} y_{j}=\lambda y_{n}, \quad n \in \mathbb{Z},
$$

for $y_{n}=\sum_{j=n+1}^{\infty} b_{j} x_{j}$ and $c_{j}=b_{j} a_{j}$. Using Cauchy-Schwarz, we have $y_{k} \rightarrow 0$ as $k \rightarrow \infty$. Using (2.17), we have

$$
y_{n}=\prod_{j=1}^{k}\left(I+c_{n+k} / \lambda\right) y_{n+k}, \quad n \in \mathbb{Z}, \quad k=1,2, \ldots
$$

Since $\left(c_{j}\right) \in \ell^{1}$, the product $\prod_{j=1}^{\infty}\left(1+\left\|c_{n+j}\right\| / \lambda\right)$ converges for each $n$. Using this in $(2.18)$ and letting $k \rightarrow \infty$, we conclude that for each $n \in \mathbb{Z}$ one has $0=y_{n}=\sum_{j=n+1}^{\infty} b_{j} x_{j}=$ $b_{n+1} x_{n+1}+y_{n+1}=b_{n+1} x_{n+1}$. Since $b_{n} x_{n}=0$, we conclude that $\lambda x_{j}=0$ for all $j$, a contradiction. Formulas (2.10), (2.11), (2.14), and (2.15) now follow from (2.2) and (2.7). To prove (2.12), represent $V_{\mathbf{a}, \mathbf{b}}^{+}=D_{\mathbf{a}} V_{\mathbf{1}, \mathbf{b}}^{+}$where $\mathbf{1}=(I)_{j \in \mathbb{Z}}$. Since $\left\|a_{j}\right\| \rightarrow 0$ as $|j| \rightarrow \infty$ and $\left\|V_{\mathbf{1}, \mathbf{b}}^{+}\right\| \leq\|\mathbf{b}\|_{\ell^{1}}$, the operator $V_{\mathbf{a}, \mathbf{b}}^{+}$is compact on $\ell^{\infty}\left(\mathbb{Z} ; \mathbb{C}^{\mathrm{d}}\right)$. The existence of nonzero $\mathbf{x} \in \ell^{\infty}$ and $\lambda$ so that $V_{\mathbf{a}, \mathbf{b}}^{+} \mathbf{x}=\lambda \mathbf{x}$ leads to a contradiction, as in (2.17) - (2.18) above, since $\left\|y_{k}\right\| \leq\|\mathbf{x}\|_{\ell \infty} \sum_{j=k+1}^{\infty}\left\|b_{k}\right\| \rightarrow 0$ as $k \rightarrow \infty$ due to $\mathbf{b} \in \ell^{1}$, and $\mathbf{c}=\left(a_{j} b_{j}\right)_{j \in \mathbb{Z}} \in \ell^{1}$.

Let $E$ denote the $(\mathrm{d} \times \mathrm{d})$ matrix having 1's on the diagonal above the main diagonal, and with zero remaining entries.

LEMMA 2.3. If $A=\lambda I+E, \lambda \in \mathbb{C}$, is a $(\mathrm{d} \times \mathrm{d})$ Jordan block, then $\left\|A^{j}\right\| \leq c|j|^{\mathrm{d}}|\lambda|^{j}$ for all $j \in \mathbb{Z}$ and some positive constant $c=c(\mathrm{~d}, \lambda)$.

Proof. Since $E^{\mathrm{d}}=0$, we have $A^{j}=\lambda^{j}\left(I+\sum_{k=1}^{\mathrm{d}-1}\left(\begin{array}{l}j \\ k\end{array}\right)(E / \lambda)^{k}\right)$ for $j>0$. The polynomial growth with $j$ of the binomial coefficients $\left(\begin{array}{l}j \\ k\end{array}\right)$ gives the result. For $j>0$, we estimate 
the norm of $A^{-j}=\lambda^{-j} \sum_{k_{1}=0}^{\mathrm{d}-1} \sum_{k_{2}=k_{1}}^{\mathrm{d}-1} \cdots \sum_{k_{j}=k_{j-1}}^{\mathrm{d}-1}(-E / \lambda)^{k_{j}}$ by $c|\lambda|^{-j} S(0, j)$, where we denote for $k \in[0, \mathrm{~d}-1]$ :

$$
S(k, j)=\sum_{k_{1}=k}^{\mathrm{d}-1} \sum_{k_{2}=k_{1}}^{\mathrm{d}-1} \cdots \sum_{k_{j}=k_{j}-1}^{\mathrm{d}-1} 1=\sum_{k_{1}=k}^{\mathrm{d}-1} S\left(k_{1}, j-1\right) .
$$

Using the formula $\sum_{k_{1}=1}^{\mathrm{d}-k} k_{1}\left(k_{1}+1\right) \ldots\left(k_{1}+j-1\right)=(\mathrm{d}-k) \ldots(\mathrm{d}-k+j) /(j+1)$ and induction, one obtains $S(k, j)=(\mathrm{d}-k)(\mathrm{d}-k+1) \ldots(\mathrm{d}-k+j-1) / j$ ! and the lemma follows.

3. Fredholm determinants. Below, we will make use of the following assumptions:

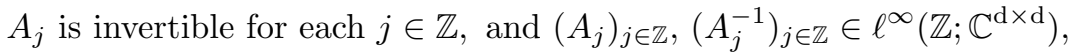
equation $x_{j+1}=A_{j} x_{j}, j \in \mathbb{Z}$, has an exponential dichotomy $P$ on $\mathbb{Z}$,

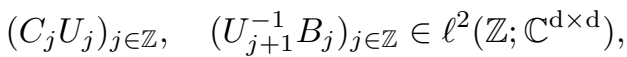
$A_{j}^{\times}=A_{j}+B_{j} C_{j}$ is invertible for each $j \in \mathbb{Z}$.

Assume (3.1). Then the fundamental matrix solution $\mathbf{U}=\left(U_{j}\right)_{j \in \mathbb{Z}}$ satisfying $U_{j+1}=$ $A_{j} U_{j}, j \in \mathbb{Z}$, and $U_{0}=I$ is given by the formulas

$$
U_{j}=A_{j-1} \cdot \ldots \cdot A_{0}, \quad U_{-j}=A_{-j}^{-1} \cdot \ldots \cdot A_{-1}^{-1}, \quad j=1,2, \ldots
$$

Similarly, assume (3.4). Then any matrix solution $\left(U_{j}^{\times}\right)_{j \in \mathbb{Z}}$ satisfying $U_{j+1}^{\times}=A_{j}^{\times} U_{j}^{\times}, j \in$ $\mathbb{Z}$, is given by the formulas

$$
U_{j}^{\times}=A_{j-1}^{\times} \cdot \ldots \cdot A_{0}^{\times} U_{0}^{\times}, \quad U_{-j}^{\times}=\left(A_{-j}^{\times}\right)^{-1} \cdot \ldots \cdot\left(A_{-1}^{\times}\right)^{-1} U_{0}^{\times}, \quad j=1,2, \ldots
$$

Here, $U_{0}^{\times}$is an arbitrary (possibly singular!) matrix. Recall that $\mathbf{U}$ has the exponential dichotomy over $\mathbb{Z}$ with the (unstable) projection $P$ provided the following inequalities hold for some $c \geq 1$ and $\alpha>0$ :

$$
\left\|U_{j}(I-P) U_{k+1}^{-1}\right\| \leq c e^{-\alpha(j-k)} \text { for } j>k, \quad\left\|U_{j} P U_{k+1}^{-1}\right\| \leq c e^{-\alpha(k-j)} \text { for } j \leq k .
$$

We let

$$
\mathrm{d}_{1}=\operatorname{dim} \operatorname{Im} P \text { and } \mathrm{d}_{2}=\operatorname{dim} \operatorname{Im}(I-P) \text { so that } \mathrm{d}_{1}+\mathrm{d}_{2}=\mathrm{d} .
$$

Under assumptions (3.1) - (3.2) the operator $\mathcal{T}$ in (1.1) is well-defined. Using notation (2.5) - (2.6), it can be written as $\mathcal{T}=V_{\mathbf{a}_{1}, \mathbf{b}_{1}}^{-}+D_{\mathbf{d}}+V_{\mathbf{a}_{2}, \mathbf{b}_{2}}^{+}$, where $\mathbf{a}_{1}=\left(C_{j} U_{j}(I-P)\right)_{j \in \mathbb{Z}}$, $\mathbf{b}_{1}=\left((I-P) U_{j+1}^{-1} B_{j}\right)_{j \in \mathbb{Z}}, \mathbf{d}=\left(-C_{j} U_{j} P U_{j+1}^{-1} B_{j}\right)_{j \in \mathbb{Z}}, \mathbf{a}_{2}=\left(-C_{j} U_{j} P\right)_{j \in \mathbb{Z}}$, and $\mathbf{b}_{2}=$ $\left(P U_{j+1}^{-1} B_{j}\right)_{j \in \mathbb{Z}}$. If assumption (3.3) holds then $\mathbf{a}_{1}, \mathbf{b}_{1}, \mathbf{a}_{2}, \mathbf{b}_{2} \in \ell^{2}$ and, using CauchySchwarz, $\mathbf{d} \in \ell^{1} \subset \ell^{2}$, so that we have $\mathcal{T} \in \mathcal{B}_{2}\left(\ell^{2}\right)$ by $(2.8)$, and $\operatorname{thus}_{\operatorname{det}_{2}}(I-\mathcal{T})$ is well-defined. Below, we will sometimes assume that $\mathcal{T} \in \mathcal{B}_{1}\left(\ell^{2}\right)$ so that $\operatorname{det}(I-\mathcal{T})$ is well-defined. Note that $\mathcal{T} \in \mathcal{B}_{1}\left(\ell^{2}\right)$ provided, say, (3.3) is replaced by the assumption $\left(C_{j} U_{j}\right)_{j \in \mathbb{Z}},\left(U_{j+1}^{-1} B_{j}\right)_{j \in \mathbb{Z}} \in \ell^{1}\left(\mathbb{Z} ; \mathbb{C}^{\mathrm{d} \times \mathrm{d}}\right)$. Indeed, under this latter assumption we have $\mathbf{a}_{1}, \mathbf{b}_{1}, \mathbf{a}_{2}, \mathbf{b}_{2}, \mathbf{d} \in \ell^{1} ;$ thus $\mathcal{T} \in \mathcal{B}_{1}\left(\ell^{2}\right)$ by $(2.13)$.

We remark that every difference operator $\mathcal{T}$ with a semi-separable kernel

$$
T_{j k}=\mathbf{a}_{2}(j) \mathbf{b}_{2}(k) \text { for } j>k \text { and } T_{j k}=\mathbf{a}_{1}(j) \mathbf{b}_{1}(k) \text { for } j \leq k,
$$


with $\mathbf{a}_{i}=\left(\mathbf{a}_{i}(j)\right)_{j \in \mathbb{Z}} \in \ell^{2}\left(\mathbb{Z} ; \mathbb{C}^{\mathrm{d} \times \mathrm{d}_{i}}\right)$ and $\mathbf{b}_{i}=\left(\mathbf{b}_{i}(j)\right)_{j \in \mathbb{Z}} \in \ell^{2}\left(\mathbb{Z} ; \mathbb{C}^{\mathrm{d}_{i} \times \mathrm{d}}\right), i=1,2$, and $\mathrm{d}_{1}+\mathrm{d}_{2}=\mathrm{d}$, can be written in the form (1.1) by setting for a fixed $\alpha>0$ and all $j \in \mathbb{Z}$ :

$$
\begin{aligned}
& P=\left[\begin{array}{cc}
0 & 0 \\
0 & I_{\mathrm{d}_{1} \times \mathrm{d}_{1}}
\end{array}\right], \quad U_{j}=\left[\begin{array}{cc}
e^{-\alpha j} I_{\mathrm{d}_{2} \times \mathrm{d}_{2}} & 0 \\
0 & e^{\alpha j} I_{\mathrm{d}_{1} \times \mathrm{d}_{1}}
\end{array}\right], \\
& C_{j}=\left[\begin{array}{lll}
e^{\alpha j} \mathbf{a}_{2}(j) & e^{-\alpha j} \mathbf{a}_{1}(j)
\end{array}\right], \quad B_{j}=\left[\begin{array}{ll}
e^{-\alpha(j+1)} \mathbf{b}_{2}(j) & e^{\alpha(j+1)} \mathbf{b}_{1}(j)
\end{array}\right]^{\top},
\end{aligned}
$$

where $T$ means transposition of the $(1 \times 2)$ block-row [. $\cdot]$.

We will use the following representations of the operator $\mathcal{T}$ defined in (1.1):

$$
\begin{aligned}
(\mathcal{T} \mathbf{x})_{j} & =\sum_{k=-\infty}^{j-1} C_{j} U_{j}(I-P) U_{k+1}^{-1} B_{k} x_{k}-\sum_{k=j}^{\infty} C_{j} U_{j} P U_{k+1}^{-1} B_{k} x_{k} \\
& =\sum_{k=-\infty}^{j-1} C_{j} U_{j} U_{k+1}^{-1} B_{k} x_{k}-\sum_{k=-\infty}^{\infty} C_{j} U_{j} P U_{k+1}^{-1} B_{k} x_{k} \\
& =-\sum_{k=j}^{\infty} C_{j} U_{j} U_{k+1}^{-1} B_{k} x_{k}+\sum_{k=-\infty}^{\infty} C_{j} U_{j}(I-P) U_{k+1}^{-1} B_{k} x_{k}, j \in \mathbb{Z} .
\end{aligned}
$$

Accordingly, we define the following operators:

$$
\begin{aligned}
& \left(H_{-} \mathbf{x}\right)_{j}=\sum_{k=-\infty}^{j-1} C_{j} U_{j} U_{k+1}^{-1} B_{k} x_{k}, \quad\left(H_{+} \mathbf{x}\right)_{j}=-\sum_{k=j}^{\infty} C_{j} U_{j} U_{k+1}^{-1} B_{k} x_{k}, \\
& (D \mathbf{x})_{j}=-C_{j} U_{j} U_{j+1}^{-1} B_{j} x_{j}, \quad\left(H_{+}^{0} \mathbf{x}\right)_{j}=-\sum_{k=j+1}^{\infty} C_{j} U_{j} U_{k+1}^{-1} B_{k} x_{k}, \\
& (Q x)_{j}=C_{j} U_{j} P x, x \in \mathbb{C}^{\mathrm{d}}, \quad R \mathbf{x}=-P \sum_{k=-\infty}^{\infty} U_{k+1}^{-1} B_{k} x_{k} \in \mathbb{C}^{\mathrm{d}_{1}}, \\
& (S x)_{j}=C_{j} U_{j}(I-P) x, x \in \mathbb{C}^{\mathrm{d}}, \quad W \mathbf{x}=(I-P) \sum_{k=-\infty}^{\infty} U_{k+1}^{-1} B_{k} x_{k} \in \mathbb{C}^{\mathrm{d}_{2}},
\end{aligned}
$$

so that $(3.11)-(3.12)$ in this notation become

$$
\begin{aligned}
\mathcal{T} & =H_{-}+Q R \\
& =H_{+}+S W=D+H_{+}^{0}+S W .
\end{aligned}
$$

We stress that the operators $R$ and $W$ have finite ranks $\mathrm{d}_{1}$ and $\mathrm{d}_{2}$, respectively. Properties of the operators (3.13) - (3.16) are summarized in the following lemmas.

Lemma 3.1. Assume (3.1) - (3.3). Then $\sigma\left(H_{-}\right)=\{0\}, H_{-} \in \mathcal{B}_{2}\left(\ell^{2}\right)$, and $\operatorname{det}_{2}\left(I-H_{-}\right)=$ 1. If, in addition to (3.1) - (3.3), we assume that $\mathcal{T} \in \mathcal{B}_{1}\left(\ell^{2}\right)$, then $H_{-} \in \mathcal{B}_{1}\left(\ell^{2}\right)$ and $\operatorname{det}\left(I-H_{-}\right)=1$.

Lemma 3.2. Assume (3.1) - (3.4). Then:

$$
D \in \mathcal{B}_{1}\left(\ell^{2}\right) \text { and } \operatorname{det}(I-D)=\prod_{j \in \mathbb{Z}}\left(\operatorname{det} A_{j}^{-1} \operatorname{det}\left(A_{j}+B_{j} C_{j}\right)\right),
$$

$$
\text { the operator }(I-D) \text { is invertible, }
$$




$$
\begin{gathered}
\operatorname{det}_{2}(I-D)=\prod_{j \in \mathbb{Z}}\left(\operatorname{det} A_{j}^{-1} \operatorname{det}\left(A_{j}+B_{j} C_{j}\right)\right) \exp \sum_{j \in \mathbb{Z}}-\operatorname{tr}\left(C_{j} U_{j} U_{j+1}^{-1} B_{j}\right), \\
\sigma\left((I-D)^{-1} H_{+}^{0}\right)=\{0\}, \quad(I-D)^{-1} H_{+}^{0} \in \mathcal{B}_{2}\left(\ell^{2}\right), \text { and } \\
\operatorname{det}_{2}\left(I-(I-D)^{-1} H_{+}^{0}\right)=1 .
\end{gathered}
$$

If, in addition to (3.1) - (3.4), we assume that $\mathcal{T} \in \mathcal{B}_{1}\left(\ell^{2}\right)$ then

$$
(I-D)^{-1} H_{+}^{0} \in \mathcal{B}_{1}\left(\ell^{2}\right), \text { and } \operatorname{det}\left(I-(I-D)^{-1} H_{+}^{0}\right)=1 .
$$

Lemma 3.3. Assume (3.1) - (3.4). Then $H_{+} \in \mathcal{B}_{2}\left(\ell^{2}\right) \operatorname{det}_{2}\left(I-H_{+}\right)=\operatorname{det}_{2}(I-D)$, and the operator $I-H_{+}$is invertible. If, in addition to (3.1) - (3.4), we assume that $\mathcal{T} \in \mathcal{B}_{1}\left(\ell^{2}\right)$ then $H_{+} \in \mathcal{B}_{1}\left(\ell^{2}\right)$ and $\operatorname{det}\left(I-H_{+}\right)=\operatorname{det}(I-D)$.

Proof. Using notation (2.5), we remark that $H_{-}=V_{\mathbf{a}, \mathbf{b}}^{-}$with $\mathbf{a}=\left(C_{j} U_{j}\right)_{j \in \mathbb{Z}}$ and $\mathbf{b}=$ $\left(U_{j+1}^{-1} B_{j}\right)_{j \in \mathbb{Z}}$. Since $\mathbf{a}, \mathbf{b} \in \ell^{2}$ by (3.3), the first three assertions in Lemma 3.1 follow, respectively, from (2.9), (2.8), and (2.10). If $\mathcal{T} \in \mathcal{B}_{1}\left(\ell^{2}\right)$ then $H_{-} \in \mathcal{B}_{1}\left(\ell^{2}\right)$ because $R \in \mathcal{B}_{1}\left(\ell^{2}\right)$ is of finite rank and (3.17) holds. We already know that $\sigma\left(H_{-}\right)=\{0\}$. Thus, $\operatorname{det}\left(I-H_{-}\right)=1$ follows from $(2.1)$, and Lemma 3.1 is proved.

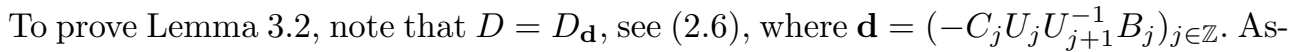
sumption (3.3) and Cauchy-Schwarz imply $\mathbf{d} \in \ell^{1}$. Then $D \in \mathcal{B}_{1}\left(\ell^{2}\right)$ by (2.13). Moreover, using (2.15) and the identity $U_{j+1}=A_{j} U_{j}, j \in \mathbb{Z}$, we verify (3.19) as follows:

$$
\begin{aligned}
\operatorname{det}(I-D) & =\prod_{j \in \mathbb{Z}} \operatorname{det}\left(I_{\mathrm{d} \times \mathrm{d}}+C_{j} U_{j} U_{j+1}^{-1} B_{j}\right)=\prod_{j \in \mathbb{Z}} \operatorname{det}\left(I+U_{j} U_{j+1}^{-1} B_{j} C_{j}\right) \\
& =\prod_{j \in \mathbb{Z}} \operatorname{det}\left(I+A_{j}^{-1} B_{j} C_{j}\right)=\prod_{j \in \mathbb{Z}} \operatorname{det} A_{j}^{-1} \operatorname{det}\left(A_{j}+B_{j} C_{j}\right) .
\end{aligned}
$$

To prove (3.20), we use assumption (3.4) and the identity $U_{j+1}=A_{j} U_{j}$ to infer:

$$
\begin{aligned}
& \left(I+C_{j} U_{j} U_{j+1}^{-1} B_{j}\right)^{-1}=\left(I+C_{j} A_{j}^{-1} B_{j}\right)^{-1}=I-C_{j}\left(I+A_{j}^{-1} B_{j} C_{j}\right)^{-1} A_{j}^{-1} B_{j} \\
& \quad=I-C_{j}\left(A_{j}+B_{j} C_{j}\right)^{-1} B_{j}=I-C_{j} U_{j}\left(U_{j+1}^{-1}\left(A_{j}+B_{j} C_{j}\right) U_{j}\right)^{-1} U_{j+1}^{-1} B_{j} \\
& \quad=I-C_{j} U_{j}\left(I+U_{j+1}^{-1} B_{j} C_{j} U_{j}\right)^{-1} U_{j+1}^{-1} B_{j} .
\end{aligned}
$$

Note that $\left(C_{j} U_{j}\right)_{j \in \mathbb{Z}},\left(U_{j+1}^{-1} B_{j}\right)_{j \in \mathbb{Z}} \in \ell^{2} \subset \ell^{\infty}\left(\mathbb{Z} ; \mathbb{C}^{\mathrm{d} \times \mathrm{d}}\right)$ and $\lim _{j \rightarrow \infty}\left\|U_{j+1}^{-1} B_{j} C_{j} U_{j}\right\|=0$ by assumption (3.3). Thus (3.20) holds and, moreover, $(I-D)^{-1}=D_{\mathbf{d}}$, where

$$
\mathbf{d}=\left(\left(I+C_{j} U_{j} U_{j+1}^{-1} B_{j}\right)^{-1}\right)_{j \in \mathbb{Z}} \in \ell^{\infty}\left(\mathbb{Z} ; \mathbb{C}^{\mathrm{d} \times \mathrm{d}}\right) .
$$

Formula (3.21) follows from (3.19) and (2.3). Using notation (2.5) and (3.24) we remark that $(I-D)^{-1} H_{+}^{0}=V_{\mathbf{a}, \mathbf{b}}^{+}$, where $\mathbf{a}=\left(-\left(I+C_{j} U_{j} U_{j+1}^{-1} B_{j}\right)^{-1} C_{j} U_{j}\right)_{j \in \mathbb{Z}}$ and $\mathbf{b}=\left(U_{j+1}^{-1} B_{j}\right)_{j \in \mathbb{Z}}$. By $\mathbf{d} \in \ell^{\infty}$ in (3.24) and assumption (3.3) we conclude $\mathbf{a}, \mathbf{b} \in$ $\ell^{2}$, and then (3.22) follows from (2.9), (2.8), and (2.10). Finally, if $\mathcal{T} \in \mathcal{B}_{1}\left(\ell^{2}\right)$ then $H_{+}=D+H_{+}^{0} \in \mathcal{B}_{1}\left(\ell^{2}\right)$ by (3.18) since $W$ is of finite rank. Since $D=D_{\mathbf{d}}$ with $\mathbf{d}=\left(-C_{j} U_{j} U_{j+1}^{-1} B_{j}\right) \in \ell^{1}$, see assumption (3.3), by (2.13) we have $D \in \mathcal{B}_{1}\left(\ell^{2}\right)$. Therefore $H_{+}^{0} \in \mathcal{B}_{1}\left(\ell^{2}\right)$ and thus $(I-D)^{-1} H_{+}^{0} \in \mathcal{B}_{1}\left(\ell^{2}\right)$ since $(I-D)^{-1}$ is a bounded operator. The last assertion in (3.23) now follows from $\sigma\left((I-D)^{-1} H_{+}^{0}\right)=\{0\}$ and (2.1). Assertions in Lemma 3.3 follow from the identity $I-H_{+}=(I-D)\left(I-(I-D)^{-1} H_{+}^{0}\right)$. 
Our first main result gives a formula for the (modified) Fredholm determinant of the (infinite-dimensional) operator $I-\mathcal{T}$ in terms of finite-dimensional determinants.

Theorem 3.4. Assume (3.1), (3.2), (3.3), and (3.4). Then

$$
\begin{aligned}
\operatorname{det}_{2}(I-\mathcal{T})= & \operatorname{det}_{\mathbb{C}^{\mathrm{d}_{1}}}\left(I_{\mathrm{d}_{1} \times \mathrm{d}_{1}}-R\left(I-H_{-}\right)^{-1} Q\right) \exp \sum_{j \in \mathbb{Z}}-\operatorname{tr}\left(P U_{j+1}^{-1} B_{j} C_{j} U_{j} P\right) \\
= & \operatorname{det}_{\mathbb{C}^{\mathrm{d}_{2}}}\left(I_{\mathrm{d}_{2} \times \mathrm{d}_{2}}-W\left(I-H_{+}\right)^{-1} S\right) \\
& \times \prod_{j \in \mathbb{Z}} \operatorname{det} A_{j}^{-1} \operatorname{det}\left(A_{j}+B_{j} C_{j}\right) \exp \sum_{j \in \mathbb{Z}}-\operatorname{tr}\left(P U_{j+1}^{-1} B_{j} C_{j} U_{j} P\right) .
\end{aligned}
$$

If, in addition to (3.1) - (3.4), we assume that $\mathcal{T} \in \mathcal{B}_{1}\left(\ell^{2}\right)$, then

$$
\begin{aligned}
\operatorname{det}(I-\mathcal{T}) & =\operatorname{det}_{\mathbb{C}^{\mathrm{d}_{1}}}\left(I_{\mathrm{d}_{1} \times \mathrm{d}_{1}}-R\left(I-H_{-}\right)^{-1} Q\right) \\
& =\operatorname{det}_{\mathbb{C}^{\mathrm{d}_{2}}}\left(I_{\mathrm{d}_{2} \times \mathrm{d}_{2}}-W\left(I-H_{+}\right)^{-1} S\right) \prod_{j \in \mathbb{Z}} \operatorname{det} A_{j}^{-1} \operatorname{det}\left(A_{j}+B_{j} C_{j}\right) .
\end{aligned}
$$

Proof. Using representation (3.17), recalling that $\operatorname{det}_{2}\left(I-H_{-}\right)=1$ by Lemma 3.1, noting that $\left(I-H_{-}\right)^{-1} Q R \in \mathcal{B}_{1} \subset \mathcal{B}_{2}$ because $R$ is of rank $\mathrm{d}_{1}$, and applying (2.4) and (2.3), we infer:

$$
\begin{aligned}
\operatorname{det}_{2} & (I-\mathcal{T})=\operatorname{det}_{2}\left[\left(I-H_{-}\right)\left(I-\left(I-H_{-}\right)^{-1} Q R\right)\right] \\
& =\operatorname{det}_{2}\left(I-H_{-}\right) \operatorname{det}_{2}\left(I-\left(I-H_{-}\right)^{-1} Q R\right) \exp \left(-\operatorname{tr}\left[H_{-}\left(I-H_{-}\right)^{-1} Q R\right]\right) \\
& =\operatorname{det}\left(I-\left(I-H_{-}\right)^{-1} Q R\right) \exp \operatorname{tr}\left[\left(I-H_{-}\right)^{-1} Q R\right] \exp \left(-\operatorname{tr}\left[H_{-}\left(I-H_{-}\right)^{-1} Q R\right]\right) \\
& =\operatorname{det}\left(I-R\left(I-H_{-}\right)^{-1} Q\right) \exp \operatorname{tr}(Q R) \\
& =\operatorname{det}_{\mathbb{C}^{\mathrm{d}_{1}}}\left(I_{\mathrm{d}_{1} \times \mathrm{d}_{1}}-R\left(I-H_{-}\right)^{-1} Q\right) \exp \operatorname{tr}(R Q) .
\end{aligned}
$$

Using (3.15), we have (3.25). To establish (3.26), we first apply representation (3.18), Lemma 3.3, and (2.4):

$$
\begin{aligned}
& \operatorname{det}_{2}(I-\mathcal{T})=\operatorname{det}_{2}\left[\left(I-H_{+}\right)\left(I-\left(I-H_{+}\right)^{-1} S W\right)\right] \\
& =\operatorname{det}_{2}\left(I-H_{+}\right) \operatorname{det}_{2}\left(I-\left(I-H_{+}\right)^{-1} S W\right) \exp \left(-\operatorname{tr}\left[H_{+}\left(I-H_{+}\right)^{-1} S W\right]\right) \\
& =\operatorname{det}_{2}(I-D) \operatorname{det}\left(I-\left(I-H_{+}\right)^{-1} S W\right) \exp \left(\operatorname{tr}\left[\left(I-H_{+}\right)^{-1} S W-H_{+}\left(I-H_{+}\right)^{-1} S W\right]\right) \\
& =\operatorname{det}_{2}(I-D) \operatorname{det}\left(I-W\left(I-H_{+}\right)^{-1} S\right) \exp \operatorname{tr}(S W) \\
& =\operatorname{det}_{2}(I-D) \operatorname{det}_{\mathbb{C}^{\mathrm{d}_{2}}}\left(I_{\mathrm{d}_{2} \times \mathrm{d}_{2}}-W\left(I-H_{+}\right)^{-1} S\right) \exp \operatorname{tr}(W S),
\end{aligned}
$$

recalling that $W$ is of rank $\mathrm{d}_{2}$, and thus $\left(I-H_{+}\right)^{-1} S W \in \mathcal{B}_{1} \subset \mathcal{B}_{2}$, which allows us to use (2.3). Using (3.21) and (3.16) we therefore have:

$$
\begin{aligned}
\operatorname{det}_{2}(I-\mathcal{T})=\operatorname{det}_{\mathbb{C}^{\mathrm{d}_{2}}}\left(I_{\mathrm{d}_{2} \times \mathrm{d}_{2}}-W\left(I-H_{+}\right)^{-1} S\right) \prod_{j \in \mathbb{Z}} \operatorname{det} A_{j}^{-1} \operatorname{det}\left(A_{j}+B_{j} C_{j}\right) \\
\times \exp \sum_{j \in \mathbb{Z}} \operatorname{tr}\left[-C_{j} U_{j} U_{j+1}^{-1} B_{j}+C_{j} U_{j}(I-P) U_{j+1}^{-1} B_{j}\right],
\end{aligned}
$$

which implies (3.26). Formula (3.27) follows from representation (3.17) and Lemma 3.1:

$$
\begin{aligned}
& \operatorname{det}(I-\mathcal{T})=\operatorname{det}\left[\left(I-H_{-}\right)\left(I-\left(I-H_{-}\right)^{-1} Q R\right)\right] \\
& \quad=\operatorname{det}\left(I-H_{-}\right) \operatorname{det}\left(I-R\left(I-H_{-}\right)^{-1} Q\right)=\operatorname{det}_{\mathbb{C}^{d_{1}}}\left(I-R\left(I-H_{-}\right)^{-1} Q\right) .
\end{aligned}
$$


Formula (3.28) follows from representation (3.18) and Lemmas 3.2 - 3.3:

$$
\begin{aligned}
& \operatorname{det}(I-\mathcal{T})=\operatorname{det}\left[\left(I-H_{+}\right)\left(I-\left(I-H_{+}\right)^{-1} S W\right)\right] \\
& \quad=\operatorname{det}\left(I-H_{+}\right) \operatorname{det}\left(I-\left(I-H_{+}\right)^{-1} S W\right)=\operatorname{det}(I-D) \operatorname{det}\left(I-W\left(I-H_{+}\right)^{-1} S\right)
\end{aligned}
$$

which concludes the proof.

Our next objective is to relate the finite-dimensional determinants in the right-hand side of $(3.25)-(3.28)$ to the determinants of a particular matrix solution $\left(\mathcal{U}_{j}^{\times}\right)_{j \in \mathbb{Z}}$ satisfying $\mathcal{U}_{j+1}^{\times}=A_{j}^{\times} \mathcal{U}_{j}^{\times}$. We stress that this solution could be singular. For this, we consider the following matrix difference equations:

$$
\begin{aligned}
& X_{j}^{+}=C_{j} U_{j}(I-P)-\sum_{k=j}^{\infty} C_{j} U_{j} U_{k+1}^{-1} B_{k} X_{k}^{+}, \\
& X_{j}^{-}=C_{j} U_{j} P+\sum_{k=-\infty}^{j-1} C_{j} U_{j} U_{k+1}^{-1} B_{k} X_{k}^{-}, \quad j \in \mathbb{Z} .
\end{aligned}
$$

Using notation (3.13) - (3.16), equations (3.29) - (3.30) for $\mathbf{X}^{ \pm}=\left(X_{j}^{ \pm}\right)_{j \in \mathbb{Z}}$ can be rewritten as follows:

$$
\mathbf{X}^{+} x=S x+H_{+} \mathbf{X}^{+} x, \quad \mathbf{X}^{-} x=Q x+H_{-} \mathbf{X}^{-} x, \quad x \in \mathbb{C}^{\mathrm{d}} .
$$

By assumption (3.3), we have $S x, Q x \in \ell^{2}\left(\mathbb{Z} ; \mathbb{C}^{\mathrm{d}}\right)$. By Lemmas 3.1 and 3.3 operators $I-$ $H_{ \pm}$are invertible. Thus, (3.29) - (3.30) have a unique pair of solutions $\mathbf{X}^{ \pm} \in \ell^{2}\left(\mathbb{Z} ; \mathbb{C}^{\mathrm{d} \times \mathrm{d}}\right)$ given by

$$
\mathbf{X}^{+}=\left(I-H_{+}\right)^{-1} S \text { and } \mathbf{X}^{-}=\left(I-H_{-}\right)^{-1} Q .
$$

Since the solutions $\mathbf{X}^{ \pm}$of (3.29) - (3.30) are unique, multiplying (3.29) by $(I-P)$ and (3.30) by $P$ from the right, we also have: $X_{j}^{+}=X_{j}^{+}(I-P)$ and $X_{j}^{-}=X_{j}^{-} P, j \in \mathbb{Z}$. Thus, we can treat matrices $X_{j}^{ \pm}$as operators $X_{j}^{+}: \operatorname{Im}(I-P) \rightarrow \mathbb{C}^{\mathrm{d}}$ and $X_{j}^{-}: \operatorname{Im} P \rightarrow \mathbb{C}^{\mathrm{d}}$. Using (3.32) and notations (3.15) - (3.16) we then have for $X_{j}^{ \pm}$from (3.29) - (3.30):

$$
\begin{array}{r}
\operatorname{det}_{\mathbb{C}^{\mathrm{d}_{1}}}\left(I_{\mathrm{d}_{1} \times \mathrm{d}_{1}}-R\left(I-H_{-}\right)^{-1} Q\right)=\operatorname{det}_{\mathbb{C}^{\mathrm{d}_{1}}}\left(I_{\mathrm{d}_{1} \times \mathrm{d}_{1}}+\sum_{k=-\infty}^{\infty} P U_{k+1}^{-1} B_{k} X_{k}^{-} P\right), \\
\operatorname{det}_{\mathbb{C}^{\mathrm{d}_{2}}}\left(I_{\mathrm{d}_{2} \times \mathrm{d}_{2}}-W\left(I-H_{+}\right)^{-1} S\right) \\
=\operatorname{det}_{\mathbb{C}^{\mathrm{d}_{2}}}\left(I_{\mathrm{d}_{2} \times \mathrm{d}_{2}}-\sum_{k=-\infty}^{\infty}(I-P) U_{k+1}^{-1} B_{k} X_{k}^{+}(I-P)\right) .
\end{array}
$$

We remark that the series $\sum_{k=-\infty}^{\infty} U_{k+1}^{-1} B_{k} X_{k}^{ \pm}$converge absolutely by assumption (3.3), $\mathbf{X}^{ \pm} \in \ell^{2}\left(\mathbb{Z} ; \mathbb{C}^{\mathrm{d} \times \mathrm{d}}\right)$, and Cauchy-Schwarz.

Using the direct sum decomposition $\mathbb{C}^{\mathrm{d}}=\operatorname{Im}(I-P) \oplus \operatorname{Im} P$, consider a matrix sequence, $\mathcal{U}^{\times}=\left(\mathcal{U}_{j}^{\times}\right)_{j \in \mathbb{Z}}$, defined by $\mathcal{U}_{j}^{\times}=U_{j} V_{j}$ where $\left(U_{j}\right)_{j \in \mathbb{Z}}$ is the fundamental matrix solution of the unperturbed equation $x_{j+1}=A_{j} x_{j}, U_{0}=I$, and the $(2 \times 2)$ block matrix $V_{j}$ is defined as follows: 


$$
V_{j}=\left[\begin{array}{cc}
I-P-\sum_{k=j}^{\infty}(I-P) U_{k+1}^{-1} B_{k} X_{k}^{+}(I-P) & \sum_{k=-\infty}^{j-1}(I-P) U_{k+1}^{-1} B_{k} X_{k}^{-} P \\
-\sum_{k=j}^{\infty} P U_{k+1}^{-1} B_{k} X_{k}^{+}(I-P) & P+\sum_{k=-\infty}^{j-1} P U_{k+1}^{-1} B_{k} X_{k}^{-} P
\end{array}\right]
$$

First, we claim that $\mathcal{U}^{\times}$is a solution of the matrix equation $\mathcal{U}_{j+1}^{\times}=A_{j}^{\times} \mathcal{U}_{j}^{\times}, j \in \mathbb{Z}$. Indeed, (3.35) and $U_{j+1}=A_{j} U_{j}$ imply:

$$
\begin{aligned}
& \mathcal{U}_{j+1}^{\times}=U_{j+1} V_{j+1}=U_{j+1} V_{j}+B_{j}\left[X_{j}^{+}(I-P) \quad X_{j}^{-} P\right] \\
& =A_{j} \mathcal{U}_{j}^{\times}+B_{j}\left[X_{j}^{+}(I-P) \quad X_{j}^{-} P\right],
\end{aligned}
$$

for the $(1 \times 2)$ block row $\left[X_{j}^{+}(I-P) \quad X_{j}^{-} P\right]: \operatorname{Im}(I-P) \oplus \operatorname{Im} P \rightarrow \mathbb{C}^{\mathrm{d}}$. Using (3.29) (3.30) and (3.35), we also have $\left[X_{j}^{+}(I-P) \quad X_{j}^{-} P\right]=C_{j} U_{j} V_{j}$, and the claim is proved. Second, we observe that there exist limits $V_{ \pm \infty}=\lim _{j \rightarrow \pm \infty} U_{j}^{-1} \mathcal{U}_{j}^{\times}$, and the operators $V_{\infty}$ and $V_{-\infty}$ are, respectively, upper- and lower-triangular matrices in the direct-sum decomposition $\mathbb{C}^{\mathrm{d}}=\operatorname{Im}(I-P) \oplus \operatorname{Im} P$. Moreover, $\operatorname{det}_{\mathbb{C}^{d}} V_{\infty}$ and $\operatorname{det}_{\mathbb{C}^{d}} V_{-\infty}$ are equal, respectively, to the right hand sides of (3.33) and (3.34). Finally, since $\mathbf{U}$ and $\mathcal{U}^{\times}$are solutions of the equations $U_{j+1}=A_{j} U_{j}$ and $\mathcal{U}_{j+1}^{\times}=A_{j}^{\times} \mathcal{U}_{j}^{\times}$, we can use formulas (3.5) - (3.6) to recalculate the right hand sides of (3.33) and (3.34) via $\operatorname{det}_{\mathbb{C}^{\mathrm{d}}} \mathcal{U}_{0}^{\times}$(recall that $\operatorname{det} U_{0}=1$ since $\left.U_{0}=I\right)$. Using Theorem 3.4, we arrive to the following result.

Theorem 3.5. Assume (3.1) - (3.4), let $X_{j}^{ \pm}$be the matrix solutions of (3.29) - (3.30), and define $\mathcal{U}^{\times}=\left(\mathcal{U}_{j}^{\times}\right)_{j \in \mathbb{Z}}$ as $\mathcal{U}_{j}^{\times}=U_{j} V_{j}$, where $V_{j}$ are given by formula (3.35). Then

$$
\operatorname{det}_{2}(I-\mathcal{T})=\left(\operatorname{det}_{\mathbb{C}^{\mathrm{d}}} \mathcal{U}_{0}^{\times}\right) \prod_{j=0}^{\infty} \frac{\operatorname{det}\left(A_{j}+B_{j} C_{j}\right)}{\operatorname{det} A_{j}} \times \exp \sum_{j \in \mathbb{Z}}-\operatorname{tr}\left(P U_{j+1}^{-1} B_{j} C_{j} U_{j} P\right) .
$$

If, in addition to (3.1) - (3.4), we assume that $\mathcal{T} \in \mathcal{B}_{1}\left(\ell^{2}\right)$, then

$$
\operatorname{det}(I-\mathcal{T})=\left(\operatorname{det}_{\mathbb{C}^{\mathrm{d}}} \mathcal{U}_{0}^{\times}\right) \prod_{j=0}^{\infty} \frac{\operatorname{det}\left(A_{j}+B_{j} C_{j}\right)}{\operatorname{det} A_{j}} .
$$

4. The Evans determinant. In this section we continue to assume that (3.1) - (3.4) hold. Because of (3.1), $\mathbf{U}$ is exponentially bounded: $\sup _{j, k \in \mathbb{Z}} e^{-\alpha(k-j)}\left\|U_{j} U_{k+1}^{-1}\right\|<\infty$ for some $\alpha \in \mathbb{R}$. This allows us to introduce the following notions related to the Bohl (or, in other terminology, general Lyapunov) exponents. For the corresponding theory in differential equations case see [DK]; also, these notions are related to the so-called Sacker-Sell, or dynamical spectrum, see [SS] and the bibliography in [CL].

If $J=\mathbb{Z}$ or $J=\mathbb{Z}^{ \pm}$, and $Q$ is a projection on $\mathbb{C}^{\mathrm{d}}$ so that $\sup _{k \in J}\left\|U_{k} Q U_{k}^{-1}\right\|<\infty$ then the upper and lower Bohl exponents, $\varkappa_{g}$ and $\varkappa_{g}^{\prime}$, are defined as follows:

$$
\begin{aligned}
& \varkappa_{g}(Q ; J)=\inf \left\{\alpha \in \mathbb{R}: \sup _{j \geq k \in J} e^{-\alpha(j-k)}\left\|U_{j} Q U_{k+1}^{-1}\right\|<\infty\right\}, \\
& \varkappa_{g}^{\prime}(Q ; J)=\sup \left\{\alpha \in \mathbb{R}: \sup _{j \leq k \in J} e^{-\alpha(j-k)}\left\|U_{j} Q U_{k+1}^{-1}\right\|<\infty\right\} .
\end{aligned}
$$

For instance, if $P$ is the dichotomy projection, cf. (3.7), then

$$
\varkappa_{g}(I-P ; \mathbb{Z})<0<\varkappa_{g}^{\prime}(P ; \mathbb{Z}) .
$$


A system $\left\{Q_{i}\right\}_{i=1}^{\mathrm{d}_{0}}$ of disjoint projections on $\mathbb{C}^{\mathrm{d}}, 1 \leq \mathrm{d}_{0} \leq \mathrm{d}$, is called an exponential splitting of order $\mathrm{d}_{0}$ if the following holds: $Q_{1}+\cdots+Q_{\mathrm{d}_{0}}=I, \sup _{k \in J}\left\|U_{k} Q_{i} U_{k}^{-1}\right\|<\infty$, and the segments $\left[\varkappa_{g}^{\prime}\left(Q_{i} ; J\right), \varkappa_{g}\left(Q_{i} ; J\right)\right]$ are all disjoint, $i=1, \ldots, \mathrm{d}_{0}$. An exponential splitting is called the finest if there is no exponential splitting of order $\mathrm{d}_{0}+1$.

Let $\left\{Q_{i}\right\}_{i=1}^{\mathrm{d}_{0}}$ denote the finest exponential splitting over $\mathbb{Z}$ for $\mathbf{U}=\left(U_{j}\right)_{j \in \mathbb{Z}}$. In what follows we assume that projections $Q_{i}$ are numbered such that $\varkappa_{g}\left(Q_{i} ; \mathbb{Z}\right)<\varkappa_{g}^{\prime}\left(Q_{i+1} ; \mathbb{Z}\right)$; we set $Q_{0}=0, Q_{\mathrm{d}_{0}+1}=I$. Since $P$ is the dichotomy projection for $\mathbf{U}$, there exists an $n \in\left\{1, \ldots, \mathrm{d}_{0}\right\}$ such that $I-P=Q_{1}+\cdots+Q_{n}$ and $P=Q_{n+1}+\cdots+Q_{\mathrm{d}_{0}}$. Thus,

$$
\begin{array}{ll}
\varkappa_{g}^{\prime}\left(Q_{i} ; \mathbb{Z}\right) \leq \varkappa_{g}\left(Q_{i} ; \mathbb{Z}\right)<0 & \text { for } i=1, \ldots, n, \text { and } \\
0<\varkappa_{g}^{\prime}\left(Q_{i} ; \mathbb{Z}\right) \leq \varkappa_{g}\left(Q_{i} ; \mathbb{Z}\right) & \text { for } i=n+1, \ldots, \mathrm{d}_{0} .
\end{array}
$$

Clearly, $\left\{Q_{i}\right\}_{i=1}^{\mathrm{d}_{0}}$ is also an exponential splitting for $\mathbf{U}$ over $\mathbb{Z}_{+}$and $\mathbb{Z}_{-}$. We denote:

$$
\begin{gathered}
\varkappa_{i}^{\prime}=\varkappa_{g}^{\prime}\left(Q_{i} ; \mathbb{Z}\right), \varkappa_{i}^{\prime \pm}=\varkappa_{g}^{\prime}\left(Q_{i} ; \mathbb{Z}_{ \pm}\right), \varkappa_{i}=\varkappa_{g}\left(Q_{i} ; \mathbb{Z}\right), \varkappa_{i}^{ \pm}=\varkappa_{g}\left(Q_{i} ; \mathbb{Z}_{ \pm}\right), i=1, \ldots, \mathrm{d}_{0}, \\
\varepsilon_{0}=\frac{1}{2} \min \left\{-\varkappa_{n}, \varkappa_{n+1}^{\prime}, \varkappa_{i}^{\prime}-\varkappa_{i-1}: i=1, \ldots, n-1, n+1, \ldots, \mathrm{d}_{0}\right\} .
\end{gathered}
$$

In this section we will use the following assumptions for the perturbation $\left(B_{j} C_{j}\right)_{j \in \mathbb{Z}}$ : There exists a $\delta \in\left(0, \varepsilon_{0}\right)$ such that

$$
\begin{gathered}
\sum_{k=0}^{\infty} e^{\left(\varkappa_{i}^{+}-\varkappa_{i}^{\prime}++\delta\right) k}\left\|B_{k} C_{k}\right\|<\infty, \text { for } i=1, \ldots, n, \\
\sum_{k=-\infty}^{0} e^{-\left(\varkappa_{i}^{-}-\varkappa_{i}^{\prime}-+\delta\right) k}\left\|B_{k} C_{k}\right\|<\infty, \text { for } i=n+1, \ldots, \mathrm{d}_{0} .
\end{gathered}
$$

Our next objective is to construct matrix solutions of the perturbed difference equation $x_{j+1}=A_{j}^{\times} x_{j}$ that are asymptotic to the solutions $\left(U_{j} Q_{i}\right)_{j \in \mathbb{Z}}$ of the unperturbed equation $x_{j+1}=A_{j} x_{j}$. For some $N \in \mathbb{N}$ consider the following $(\mathrm{d} \times \mathrm{d})$ matrix difference equations:

$$
\begin{aligned}
& Y_{j}^{(i)}-U_{j} Q_{i}=-\sum_{k=j}^{\infty} U_{j}\left(Q_{i}+\cdots+Q_{\mathrm{d}_{0}}\right) U_{k+1}^{-1} B_{k} C_{k} Y_{k}^{(i)} \\
&+\sum_{k=N}^{j-1} U_{j}\left(I-\left(Q_{i}+\cdots+Q_{\mathrm{d}_{0}}\right)\right) U_{k+1}^{-1} B_{k} C_{k} Y_{k}^{(i)}, j>N, i=1, \ldots, n, \\
&(4.10) \sum_{k=-\infty}^{j-1} U_{j}\left(Q_{1}+\cdots+Q_{i}\right) U_{k+1}^{-1} B_{k} C_{k} Y_{k}^{(i)}-U_{j} Q_{i}= \\
&-\sum_{k=j}^{-N} U_{j}\left(I-\left(Q_{1}+\cdots+Q_{i}\right)\right) U_{k+1}^{-1} B_{k} C_{k} Y_{k}^{(i)}, j \leq-N, i=n+1, \ldots, \mathrm{d}_{0} . \\
&(4.11) \quad
\end{aligned}
$$

REMARK 4.1. If the sequence $\mathbf{Y}^{(i)}=\left(Y_{j}^{(i)}\right)$, where $j>N$, resp. $j \leq-N$, is a solution of (4.10), resp. (4.11), then this sequence is a solution of the (perturbed) difference equation

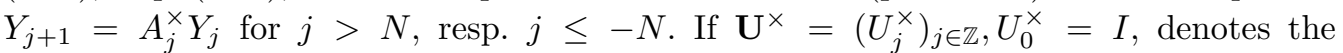
fundamental matrix solution of the equation $U_{j+1}^{\times}=A_{j}^{\times} U_{j}^{\times}$(this solution is non-singular 
by assumption (3.4)), then the solutions $\mathbf{Y}^{(i)}=\left(Y_{j}^{(i)}\right), i=1, \ldots, n$, resp. $i=n+1, \ldots, \mathrm{d}_{0}$, originally given for $j>N$, resp. $j \leq-N$, could be extended to $\mathbb{Z}_{+}=\{0,1, \ldots\}$, resp. $\mathbb{Z}_{-}=\{\ldots,-1,0\}$, by setting

$$
\begin{aligned}
& Y_{j}^{(i)}=U_{j}^{\times}\left(U_{N+1}^{\times}\right)^{-1} Y_{N+1}^{(i)}, \quad j=0, \ldots, N, \\
& Y_{j}^{(i)}=U_{j}^{\times}\left(U_{-N}^{\times}\right)^{-1} Y_{-N}^{(i)}, \quad j=-N+1, \ldots, 0 .
\end{aligned}
$$

Lemma 4.2. Assume (4.8), resp. (4.9). Then, for a sufficiently large $N=N(\delta)$, there exists a solution $\mathbf{Y}^{(i)}=\left(Y_{j}^{(i)}\right)_{j>N}, i=1, \ldots, n$, of $(4.10)$, resp. $\mathbf{Y}^{(i)}=\left(Y_{j}^{(i)}\right)_{j \leq-N}$, $i=n+1, \ldots, \mathrm{d}_{0}$, of (4.11). Moreover, these solutions satisfy $Y_{j}^{(i)}=Y_{j}^{(i)} Q_{i}$ and have the following properties:

(a)

$$
\begin{aligned}
& \sup _{j>N} e^{-\left(\varkappa_{i}^{+}+\frac{\delta}{2}\right) j}\left\|Y_{j}^{(i)}\right\|<\infty, \quad i=1, \ldots, n, \\
& \sup _{j \leq-N} e^{-\left(\varkappa_{i}^{\prime-}-\frac{\delta}{2}\right) j}\left\|Y_{j}^{(i)}\right\|<\infty, \quad i=n+1, \ldots, \mathrm{d}_{0} ;
\end{aligned}
$$

(b)

$$
\begin{aligned}
& \inf _{j>N} e^{-\left(\varkappa_{i}^{\prime+}-\frac{\delta}{2}\right) j}\left\|Y_{j}^{(i)}\right\|_{\bullet}>0, \quad i=1, \ldots, n, \\
& \inf _{j \leq-N} e^{-\left(\varkappa_{i}^{-}+\frac{\delta}{2}\right) j}\left\|Y_{j}^{(i)}\right\|_{\bullet}>0, \quad i=n+1, \ldots, \mathrm{d}_{0} ;
\end{aligned}
$$

(c)

$$
\begin{aligned}
& \sup _{j>N} e^{-\left(\varkappa_{i}^{\prime}+-\frac{\delta}{2}\right) j}\left\|Y_{j}^{(i)}-U_{j} Q_{i}\right\|<\infty, \quad i=1, \ldots, n, \\
& \sup _{j \leq-N} e^{-\left(\varkappa_{i}^{-}+\frac{\delta}{2}\right) j}\left\|Y_{j}^{(i)}-U_{j} Q_{i}\right\|<\infty, \quad i=n+1, \ldots, \mathrm{d}_{0} .
\end{aligned}
$$

Proof. For $i=1, \ldots, n$ set $\alpha=\varkappa_{i}^{\prime+}-\delta / 2$ so that $\alpha \in\left(\varkappa_{i-1}^{+}, \varkappa_{i}^{\prime+}\right)$. Using (4.1) - (4.2) with $J=\mathbb{Z}_{+}$, find constants $c_{\alpha}$ and $c_{\alpha}^{\prime}$ such that

$$
\begin{aligned}
\left\|U_{j}\left(I-\left(Q_{i}+\cdots+Q_{\mathrm{d}_{0}}\right)\right) U_{k+1}^{-1}\right\| \leq c_{\alpha} e^{\alpha(j-k)}, & j \geq k \geq 0, \\
\left\|U_{j}\left(Q_{i}+\cdots+Q_{\mathrm{d}_{0}}\right) U_{k+1}^{-1}\right\| \leq c_{\alpha}^{\prime} e^{\alpha(j-k)}, & k \geq j \geq 0 .
\end{aligned}
$$

Using assumption (4.8), choose $N$ so large that

$$
q:=\sum_{k=N}^{\infty} \max \left\{c_{\alpha}, c_{\alpha}^{\prime}\right\} e^{\left(\varkappa_{i}^{+}-\varkappa_{i}^{\prime+}+\delta\right) k}\left\|B_{k} C_{k}\right\|<1 .
$$

With this $N$, and letting $\beta=\varkappa_{i}^{+}+\delta / 2$, we define a Banach space, $\ell_{+, \beta}^{\infty}$, of $\mathbb{C}^{\mathrm{d} \times \mathrm{d}}$-valued sequences $\mathbf{u}=\left(u_{j}\right)_{j>N}$ as follows: $\ell_{+, \beta}^{\infty}=\left\{\mathbf{u}:\|\mathbf{u}\|_{+, \beta}:=\sup _{j>N} e^{-\beta j}\left\|u_{j}\right\|<\infty\right\}$. Let $T$ denote the operator corresponding to the right-hand side of (4.10), so that this equation becomes $\mathbf{Y}^{(i)}-\left(U_{j} Q_{i}\right)_{j>N}=T \mathbf{Y}^{(i)}$. We claim that $T$ is a contraction in $\ell_{+, \beta}^{\infty}$. Indeed, using (4.13) - (4.14), and then (4.15), we infer:

$$
\|T \mathbf{u}\|_{+, \beta} \leq\|\mathbf{u}\|_{+, \beta} \sup _{j>N} e^{-\beta j}\left(\sum_{k=j}^{\infty} c_{\alpha}^{\prime} e^{\alpha(j-k)}\left\|B_{k} C_{k}\right\| e^{\beta k}+\sum_{k=N}^{j-1} c_{\alpha} e^{\alpha(j-k)}\left\|B_{k} C_{k}\right\| e^{\beta k}\right)
$$

$$
\leq\|\mathbf{u}\|_{+, \beta} \max \left\{c_{\alpha}, c_{\alpha}^{\prime}\right\} \sup _{j>N} \sum_{k=N}^{\infty} e^{(\alpha-\beta)(j-k)}\left\|B_{k} C_{k}\right\|<q\|\mathbf{u}\|_{+, \beta},
$$

because $(\alpha-\beta) j=-\left(\varkappa_{i}^{+}-\varkappa_{i}^{+}+\delta\right) j<0$. Since $\sup _{j \geq 0} e^{-\beta j}\left\|U_{j} Q_{i}\right\|<\infty$ by (4.1) with $J=\mathbb{Z}_{+}, k=-1$, and $\beta>\varkappa_{i}^{+}$, we have $\left(U_{j} Q_{i}\right)_{j>N} \in \ell_{+, \beta}^{\infty}$. This proves the existence and 
uniqueness of a solution $\mathbf{Y}^{(i)} \in \ell_{+, \beta}^{\infty}$ of (4.10), and thus (a). Also, multiplying (4.10) by $Q_{i}$ from the right, and using the uniqueness, we have $Y_{j}^{(i)}=Y_{j}^{(i)} Q_{i}$. Similarly to estimate (4.16), we have

$$
\|T \mathbf{u}\|_{+, \alpha} \leq\|\mathbf{u}\|_{+, \beta} \max \left\{c_{\alpha}, c_{\alpha}^{\prime}\right\} \sum_{k=N}^{\infty} e^{(\beta-\alpha) k}\left\|B_{k} C_{k}\right\|=q\|\mathbf{u}\|_{+, \beta} .
$$

Since $\mathbf{Y}^{(i)}-\left(U_{j} Q_{i}\right)_{j>N}=T \mathbf{Y}^{(i)}$ and $\mathbf{Y}^{(i)} \in \ell_{+, \beta}^{\infty}$, we have $\mathbf{Y}^{(i)}-\left(U_{j} Q_{i}\right)_{j>N} \in \ell_{+, \alpha}^{\infty}$ and assertion $(c)$ in the lemma follows. To prove assertion $(b)$, we estimate

$$
\begin{aligned}
\left\|Y_{j}^{(i)}\right\|_{\bullet} & =\left\|Y_{j}^{(i)}-U_{j} Q_{i}+U_{j} Q_{i}\right\|_{\bullet} \geq\left\|U_{j} Q_{i}\right\|_{\bullet}-\left\|Y_{j}^{(i)}-U_{j} Q_{i}\right\| \\
& \geq\left\|U_{j} Q_{i}\right\|_{\bullet}-c_{1} e^{\alpha j}
\end{aligned}
$$

with some positive constant $c_{1}$ from $(c)$. If $\|x\|=1$ and $Q_{i} x=x$ then, using (4.2) for $J=\mathbb{Z}_{+}$, and that $\alpha+\delta / 4=\varkappa_{j}^{+}-\delta / 4<\varkappa_{j}^{\prime+}$, we infer for any $k>0$ :

$$
\begin{aligned}
1 & =\left\|Q_{i} x\right\| \leq\left\|Q_{i} U_{k+1}^{-1}\right\| \cdot\left\|U_{k+1}^{-1} Q_{i} x\right\|=\left(e^{(\alpha+\delta / 4) k}\left\|Q_{i} U_{k+1}^{-1}\right\|\right)\left(e^{-(\alpha+\delta / 4) k}\left\|U_{k+1}^{-1} Q_{i}\right\|\right) \\
& \leq\left(\sup _{0 \leq j \leq k} e^{-(\alpha+\delta / 4)(j-k)}\left\|U_{j} Q_{i} U_{k+1}^{-1}\right\|\right)\left(e^{-(\alpha+\delta / 4) k}\left\|U_{k+1}^{-1} Q_{i} x\right\|\right) \\
& \leq c e^{-(\alpha+\delta / 4) k}\left\|U_{k+1}^{-1} Q_{i} x\right\| .
\end{aligned}
$$

This implies that $\left\|U_{j} Q_{i}\right\|_{\bullet} \geq c_{2} e^{(\alpha+\delta / 4) j}$ for all $j \in \mathbb{Z}_{+}$and some $c_{2}>0$. Using (4.17), we have $e^{-\alpha j}\left\|Y_{j}^{(i)}\right\|_{\bullet} \geq c_{2} e^{(\delta / 4) j}-c_{1} \geq 1$ starting from some sufficiently large $j_{0}>N$. Since $\mathbf{Y}^{(i)}=\mathbf{Y}^{(i)} Q_{i}$ is a solution of the equation $Y_{j+1}^{(i)}=A_{j}^{\times} Y_{j}^{(i)}$, the last assertion and assumption (3.4) imply that $\left\|Y_{j}^{(i)}\right\|_{\bullet}>0$ for each $j \in\left[N, j_{0}\right]$. This proves $(b)$, and the proof of the lemma for $i=1, \ldots, n$ is complete. The proof for $i=n+1, \ldots, \mathrm{d}_{0}$ is similar.

REMARK 4.3. By Lemma 4.2 and Remark 4.1 we thus have matrix solutions $\mathbf{Y}^{(i)}=$ $\left(Y_{j}^{(i)}\right)_{j \geq 0}, i=1, \ldots, n$, resp. $\mathbf{Y}^{(i)}=\left(Y_{j}^{(i)}\right)_{j \leq 0}, i=n+1, \ldots, \mathrm{d}_{0}$, of the perturbed equation $x_{j+1}=A_{j}^{\times} x_{j}$ on $\mathbb{Z}_{+}$, resp. $\mathbb{Z}_{-}$. Define $\mathbf{Y}^{+}=\mathbf{Y}^{(1)}+\cdots+\mathbf{Y}^{(n)}$ and $\mathbf{Y}^{-}=\mathbf{Y}^{(n+1)}+\cdots+$ $\mathbf{Y}^{\left(\mathrm{d}_{0}\right)}$. Using the property $Y_{j}^{(i)} Q_{i}=Y_{j}^{(i)}$, we may view $Y_{j}^{+}$, resp. $Y_{j}^{-}$, as operators acting from $\operatorname{Im}(I-P)=\operatorname{Im} Q_{1} \oplus \cdots \oplus \operatorname{Im} Q_{n}$, resp. $\operatorname{Im} P=\operatorname{Im} Q_{n+1} \oplus \cdots \oplus \operatorname{Im} Q_{\mathrm{d}_{0}}$, to $\mathbb{C}^{\mathrm{d}}$ and thus write $\mathbf{Y}^{+}=\left[\mathbf{Y}^{(1)} \cdots \mathbf{Y}^{(n)}\right]$, resp. $\mathbf{Y}^{-}=\left[\mathbf{Y}^{(n+1)} \cdots \mathbf{Y}^{\left(\mathrm{d}_{0}\right)}\right]$ either as $(1 \times n)$-, resp. $\left(1 \times\left(\mathrm{d}_{0}-n\right)\right)$ block rows, or, cf. $(3.8)$, as $\left(\mathrm{d} \times \mathrm{d}_{2}\right)$-, resp. $\left(\mathrm{d} \times \mathrm{d}_{1}\right)$-matrices.

Assertions $(a)$ and $(b)$ of Lemma 4.2 and Remark 4.1 show that the solution $\mathbf{Y}^{(i)}$ of the perturbed equation $x_{j+1}=A_{j}^{\times} x_{j}, j \in \mathbb{Z}_{+}$, resp. $\mathbb{Z}_{-}$, corresponds to the segment $\left[\varkappa_{i}^{\prime+}, \varkappa_{i}^{+}\right]$, resp. $\left[\varkappa_{i}^{\prime-}, \varkappa_{i}^{-}\right]$, in the Bohl spectrum for $i=1, \ldots, n$, resp. $i=n+1, \ldots, \mathrm{d}_{0}$. In particular, for $i=1, \ldots, n$ one has $\left\|Y_{j}^{(i)}\right\| \rightarrow 0$ as $j \rightarrow \infty$, and for $i=n+1, \ldots, \mathrm{d}_{0}$ one has $\left\|Y_{j}^{(i)}\right\| \rightarrow 0$ as $j \rightarrow-\infty$, see (4.4) - (4.5) and recall that $\varkappa_{i}^{\prime} \leq \varkappa_{i}^{\prime} \leq \varkappa_{i}^{ \pm} \leq \varkappa_{i}$ by $(4.1)-(4.2)$. Assertion $(c)$ shows that the solutions $\mathbf{Y}^{(i)}$ of the perturbed equation are asymptotic to the solutions $\left(U_{j} Q_{i}\right)_{j \in \mathbb{Z}}$ of the unperturbed equation $x_{j+1}=A_{j} x_{j}$, $j \in \mathbb{Z}$, as $j \rightarrow \infty$ for $i=1, \ldots, n$, resp. $j \rightarrow-\infty$ for $i=n+1, \ldots, \mathrm{d}_{0}$. This motivates the following definition.

Definition 4.4. If $\mathbf{Y}^{(i)}=\left(Y_{j}^{(i)}\right)_{j \geq 0}, i=1, \ldots, n$ and $\mathbf{Y}^{(i)}=\left(Y_{j}^{(i)}\right)_{j \leq 0}, i=n+1, \ldots, \mathrm{d}_{0}$ are the matrix solutions of the perturbation $x_{j+1}=A_{j}^{\times} x_{j}, A_{j}^{\times}=A_{j}+B_{j} C_{j}$, of the 
difference equation $x_{j+1}=A_{j} x_{j}$, then the Evans determinant, $\mathcal{E}$, is defined as follows: (4.18) $\mathcal{E}=\operatorname{det}\left[Y_{0}^{+}+Y_{0}^{-}\right]$, where $Y_{0}^{+}=Y_{0}^{(1)}+\cdots+Y_{0}^{(n)}$ and $Y_{0}^{-}=Y_{0}^{(n+1)}+\cdots+Y_{0}^{\left(\mathrm{d}_{0}\right)}$.

The terminology is related to the so-called Evans function, a powerful tool frequently used for detecting isolated eigenvalues of differential (and difference) operators that appear after linearizing nonlinear equations about such special solutions as travelling waves, see $[\mathrm{AGJ}, \mathrm{JK}, \mathrm{S}]$ and $[\mathrm{BCK}, \mathrm{KK}]$, and the bibliographies therein. The Evans function, $\mathcal{D}(z)$, is usually defined, cf. [S], in the situation when the coefficients of the unperturbed and perturbed equations depend (analytically) on a (spectral) parameter $z$. Thus, in our terminology, the values of the Evans function for fixed $z$ 's are called the Evans determinants. In Proposition 4.5 we will show that the definition of the Evans determinant $\mathcal{E}$ given in (4.18) coincides with the definition of the Evans determinant $\mathcal{D}$ standardly accepted in the literature on the Evans function, cf. [JK, S]; moreover, the Evans determinant $\mathcal{E}$ gives a canonical choice among the standard Evans determinants $\mathcal{D}$ whose definition in $[\mathrm{JK}, \mathrm{S}]$ is not unique.

Out of several available (equivalent) standard definitions of $\mathcal{D}$ we chose the definition using the exponential dichotomies on $\mathbb{Z}_{+}$and $\mathbb{Z}_{-}$, cf. [S, Def. 4.1] for the differential equations case. Recall the definition from [S]. Assume that the perturbed equation $x_{j+1}=$ $A_{j}^{\times} x_{j}$ has exponential dichotomies $P_{+}$and $P_{-}$on $\mathbb{Z}_{+}$and $\mathbb{Z}_{-}$, respectively, so that for its solution $\mathbf{x}=\left(x_{j}\right)$ one has: $\left\|x_{j}\right\| \rightarrow 0$ as $j \rightarrow \infty$ if and only if $x_{0} \in \operatorname{Im}\left(I-P_{+}\right) ;\left\|x_{j}\right\| \rightarrow \infty$ as $j \rightarrow \infty$ if and only if $x_{0}$ has a nonzero component in $\operatorname{Im} P_{+} ;\left\|x_{j}\right\| \rightarrow 0$ as $j \rightarrow-\infty$ if and only if $x_{0} \in \operatorname{Im} P_{-}$; and $\left\|x_{j}\right\| \rightarrow \infty$ as $j \rightarrow-\infty$ if and only if $x_{0}$ has a nonzero component in $\operatorname{Im}\left(I-P_{-}\right)$. In addition, following $[\mathrm{S}]$, assume that $\operatorname{dim} \operatorname{Im}\left(I-P_{+}\right)=\operatorname{dim} \operatorname{Im}\left(I-P_{-}\right)$ and denote the common value of these dimensions $\mathrm{d}^{\prime}$. Choose ordered bases $u_{1}, \ldots, u_{\mathrm{d}^{\prime}}$ and $u_{\mathrm{d}^{\prime}+1}, \ldots, u_{\mathrm{d}}$ of $\operatorname{Im}\left(I-P_{+}\right)$and $\operatorname{Im} P_{-}$, respectively, and define

$$
\mathcal{D}=\operatorname{det}_{\mathbb{C}^{d}}\left[u_{1} \cdots u_{\mathrm{d}}\right]
$$

the Evans determinant. Note that $\mathcal{D}$ depends on the choice of the basis vectors $u_{i}$; however, if $\widetilde{\mathcal{D}}$ is the determinant corresponding to another choice of $\tilde{u}_{i}$, then $\mathcal{D}=c \widetilde{\mathcal{D}}$ for a nonzero $c$; if $\mathbf{A}^{\times}=\mathbf{A}^{\times}(z)$ and $u_{i}=u_{i}(z)$ and $\tilde{u}_{i}=\tilde{u}_{i}(z)$ depend on $z$ analytically then one can show $[\mathrm{S}]$ that $c=c(z)$ is a nonvanishing analytic multiplier.

Proposition 4.5. Assume (3.1), (3.2), (3.4), and (4.8) - (4.9). Then the Evans determinant $\mathcal{E}$, as defined in (4.18), coincides with $\mathcal{D}$, as defined in (4.19), where $u_{1}, \ldots u_{\mathrm{d}^{\prime}}$ and $u_{\mathrm{d}^{\prime}+1}, \ldots, u_{\mathrm{d}}$ are the columns of the matrices $Y_{0}^{+}$and $Y_{0}^{-}$, respectively.

Proof. First, we claim that

$$
\operatorname{dim} \operatorname{Im}(I-P)=\operatorname{dim} \operatorname{Im}\left(I-P_{+}\right) \text {and } \operatorname{dim} \operatorname{Im} P=\operatorname{dim} \operatorname{Im} P_{-}
$$

for the dichotomy projection $P$ over $\mathbb{Z}$ of the unperturbed equation $x_{j+1}=A_{j} x_{j}$ and the dichotomy projections $P_{ \pm}$over $\mathbb{Z}_{ \pm}$of the perturbed equation $y_{j+1}=A_{j}^{\times} y_{j}$. To prove the first equality in (4.20) (the second is proved similarly), we recall that the perturbation $B_{j} C_{j}=A_{j}^{\times}-A_{j}, j \in \mathbb{Z}$, is assumed to satisfy $\left(B_{j} C_{j}\right)_{j \in \mathbb{Z}_{+}} \in \ell^{1}\left(\mathbb{Z}_{+} ; \mathbb{C}^{\mathrm{d} \times \mathrm{d}}\right)$, cf. (4.8). Under this assumption, following the proof of [Co, Prop. 4.3], one can see that the (bounded on $\left.\mathbb{Z}_{+}\right)$solutions $\mathbf{x}=\left(x_{j}\right)_{j \in \mathbb{Z}_{+}}$of the equation $x_{j+1}=A_{j} x_{j}$ and $\mathbf{y}=\left(y_{j}\right)_{j \in \mathbb{Z}}$ of the 
equation $y_{j+1}=A_{j}^{\times} y_{j}$ are in one-to-one correspondence. Indeed, using (3.7), choose $N$ so large that the operator $T$ defined by

$$
(T \mathbf{y})_{j}=-\sum_{k=j}^{\infty} U_{j} P U_{k+1}^{-1} B_{k} C_{k} y_{k}+\sum_{k=N}^{j-1} U_{j}(I-P) U_{k+1}^{-1} B_{k} C_{k} y_{k}, \quad j \in \mathbb{Z}_{+},
$$

is a contraction on $\ell^{\infty}\left(\mathbb{Z}_{+} ; \mathbb{C}^{\mathrm{d} \times \mathrm{d}}\right)$, cf. the proof of Lemma 4.2. Then the above-mentioned correspondence is given by the formula $\mathbf{y}=\mathbf{x}+T \mathbf{y}$. Since the solutions $\mathbf{x}$ and $\mathbf{y}$ are bounded on $\mathbb{Z}_{+}$if and only if $x_{0} \in \operatorname{Im}(I-P)$, resp. $y_{0} \in \operatorname{Im}\left(I-P_{+}\right)$, and using notation (3.8), the equality $\mathrm{d}_{2}=\mathrm{d}^{\prime}$ in (4.20) follows.

Next, we claim that for the solutions $\mathbf{Y}^{+}=\left[\mathbf{Y}^{(1)} \cdots \mathbf{Y}^{(n)}\right]$ and $\mathbf{Y}^{-}=\left[\mathbf{Y}^{(n+1)} \cdots \mathbf{Y}^{\left(\mathrm{d}_{0}\right)}\right]$, see Remark 4.3, of the equation $y_{j+1}=A_{j}^{\times} y_{j}$, obtained in Lemma 4.2 and Remark 4.1, one has the following equalities:

$$
\operatorname{rank} Y_{0}^{+}=\operatorname{dim} \operatorname{Im}\left(I-P_{+}\right) \text {and } \operatorname{rank} Y_{0}^{-}=\operatorname{dim} \operatorname{Im} P_{-} .
$$

As soon as (4.21) is proved, the equality $\mathcal{E}=\mathcal{D}$ follows. Indeed, recalling (4.4) - (4.5), and the inequality $\delta<\varepsilon_{0}$ for $\varepsilon_{0}$ defined in (4.7), we use Lemma 4.2(a) to observe that $\left\|Y_{j}^{(i)}\right\| \rightarrow 0$ as $j \rightarrow \infty$, resp. $j \rightarrow-\infty$, for $i=1, \ldots, n$, resp. $i=n+1 \ldots, \mathrm{d}_{0}$. Therefore, recalling (4.20), the definitions of the exponential dichotomies $P_{ \pm}$, see (3.7), and Remark 4.3, we observe that the columns $u_{1}, \ldots, u_{\mathrm{d}_{2}}$ and $u_{\mathrm{d}_{2}+1}, \ldots, u_{\mathrm{d}}$ of the matrices $Y_{0}^{+}$and, respectively, $Y_{0}^{-}$belong to the subspace $\operatorname{Im}\left(I-P_{+}\right)$, respectively, to the subspace $\operatorname{Im} P_{-}$. By (4.21), these columns form bases in the respective subspaces, and thus $\mathcal{E}=\mathcal{D}$.

To prove the first equality in (4.21) (the second is proved similarly), we will use (4.20) and will show that $\operatorname{rank} Y_{0}^{+}=\operatorname{dim} \operatorname{Im}(I-P)$. For this, it is enough to check that $\operatorname{rank} Y_{j}^{+}=\operatorname{dim} \operatorname{Im}(I-P)$ for sufficiently large $j$ because $\operatorname{rank} Y_{j}^{+}=\operatorname{rank} Y_{0}^{+}$using $Y_{j}^{+}=U_{j}^{\times} Y_{0}^{+}$for the invertible by (3.4) matrices $U_{j}^{\times}$forming the fundamental matrix solution $\mathbf{U}^{\times}$of the equation $y_{j+1}=A_{j}^{\times} y_{j}$. Finally, since matrices $U_{j}$ are also invertible, and $I-P=Q_{1}+\cdots+Q_{n}$, we have:

$\operatorname{dim} \operatorname{Im}(I-P)=\operatorname{rank}\left[Q_{1} \cdots Q_{n}\right]=\operatorname{rank}\left[U_{j} Q_{1} \cdots U_{j} Q_{n}\right]=\operatorname{rank}\left[Y_{j}^{(1)} \cdots Y_{j}^{(n)}\right]=\operatorname{rank} Y_{j}^{+}$, where the relation $\left\|Y_{j}^{(i)}-U_{j} Q_{i}\right\| \rightarrow 0$ as $j \rightarrow \infty, i=1, \ldots, n$, from Lemma 4.2 (c) has been used to justify the third equality. Thus, (4.21) follows, and $\mathcal{E}=\mathcal{D}$ is proved.

One advantage of Definition 4.4 adapted in the current paper is that the Evans determinant given in (4.18) is uniquely defined by $\mathbf{A}$ and $\mathbf{A}^{\times}$(indeed, the finest exponential splitting $\left\{Q_{i}\right\}_{i=1}^{\mathrm{d}_{0}}$ is uniquely defined; although the solutions $\mathbf{Y}^{(i)}$ depend on $N$, see Lemma 4.2, the determinant (4.18) is proved below to be $N$-independent, see (4.22)). In other words, the columns of the matrices $Y_{0}^{+}$and $Y_{0}^{-}$give the canonical choice of the bases in $\operatorname{Im}\left(I-P_{+}\right)$and $\operatorname{Im} P_{-}$needed in (4.19). Also, if $\mathbf{A}=\mathbf{A}(z)$ and $\mathbf{A}^{\times}=\mathbf{A}^{\times}(z)$ depend on $z$ analytically, then the analyticity of the Evans function $\mathcal{E}=\mathcal{E}(z)$, where the Evans determinant $\mathcal{E}(z)$ is defined for each fixed $z$ using $\mathbf{A}(z)$ and $\mathbf{A}^{\times}(z)$ as indicated in (4.18), follows automatically from the analyticity of the corresponding Fredholm determinant whose connection to the Evans determinant is given next.

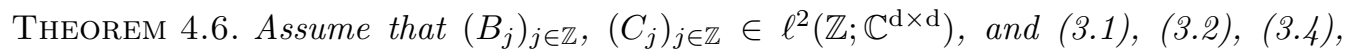
(4.8), (4.9) hold. Then the Fredholm determinant for the operator $\mathcal{T} \in \mathcal{B}_{1}\left(\ell^{2}\left(\mathbb{Z} ; \mathbb{C}^{\mathrm{d} \times \mathrm{d}}\right)\right)$, 
defined in (1.1), and the Evans determinant (4.18) are related as follows:

$$
\operatorname{det}(I-\mathcal{T})=\mathcal{E} \prod_{j=0}^{\infty} \frac{\operatorname{det}\left(A_{j}+B_{j} C_{j}\right)}{\operatorname{det} A_{j}}
$$

REMARK 4.7. In this paper we study the perturbed equation $x_{j+1}=\left(A_{j}+B_{j} C_{j}\right) x_{j}$ with the perturbation term $R_{j}=B_{j} C_{j}$ having a predefined factorization. Given a perturbed equation $x_{j+1}=\left(A_{j}+R_{j}\right) x_{j}$, we can define the factorization $R_{j}=B_{j} C_{j}$ by using the polar decomposition $R_{j}=V_{R_{j}}\left|R_{j}\right|$ and setting $B_{j}=V_{R_{j}}\left|R_{j}\right|^{\frac{1}{2}}$ and $C_{j}=\left|R_{j}\right|^{\frac{1}{2}}$. If (4.8) -

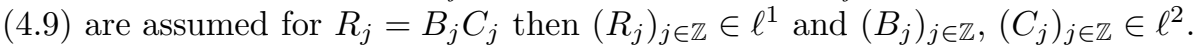

Proof. First, we remark that $\mathcal{T} \in \mathcal{B}_{1}\left(\ell^{2}\right)$ provided $\mathbf{b}=\left(B_{j}\right)_{j \in \mathbb{Z}}$ and $\mathbf{c}=\left(C_{j}\right)_{j \in \mathbb{Z}}$ belong to $\ell^{2}$. Indeed, let $\mathcal{K},(\mathcal{K} \mathbf{x})_{j}=\sum_{k \in \mathbb{Z}} K_{j k} x_{k}$, denote the (bounded on $\ell^{2}$ ) operator with the kernel given by (1.2) and satisfying $\left\|K_{j k}\right\| \leq c e^{-\alpha|k-j|}$, see (3.7). Then $\mathcal{T}=D_{\mathbf{c}} \mathcal{K} D_{\mathbf{b}}$ using notation (2.6). By (2.8) we have $D_{\mathbf{c}}, D_{\mathbf{b}} \in \mathcal{B}_{2}\left(\ell^{2}\right)$ and thus $\mathcal{T} \in \mathcal{B}_{1}$, see, e.g. [GGK, Lem. IV.7.2]. Next, we remark that the product in the right-hand side of (4.22) converges absolutely provided (3.1) and $\mathbf{b}, \mathbf{c} \in \ell^{2}$ hold:

$\prod_{j=0}^{\infty}\left|\frac{\operatorname{det}\left(A_{j}+B_{j} C_{j}\right)}{\operatorname{det} A_{j}}\right|=\prod_{j=0}^{\infty}\left|\operatorname{det}\left(I+A_{j}^{-1} B_{j} C_{j}\right)\right| \leq \prod_{j=0}^{\infty}\left(1+\mathrm{d}\left\|\left(A_{j}^{-1}\right)_{j \in \mathbb{Z}}\right\|_{\ell^{\infty}}\left\|B_{j} C_{j}\right\|\right)<\infty$ because $\left(B_{j} C_{j}\right)_{j \in \mathbb{Z}} \in \ell^{1}$.

Further, we claim that it is enough to prove (4.22) for finitely supported $\mathbf{b}$ and $\mathbf{c}$. For $M \in \mathbb{N}$ let $\chi_{M}$ denote the characteristic function of $[-M, M] \cap \mathbb{Z}$, and set $\mathbf{b}^{(M)}=$ $\left(\chi_{M}(j) B_{j}\right)_{j \in \mathbb{Z}}, \mathbf{c}^{(M)}=\left(\chi_{M}(j) C_{j}\right)_{j \in \mathbb{Z}}, \mathcal{T}^{(M)}=D_{\mathbf{c}^{(M)}} \mathcal{K} D_{\mathbf{b}^{(M)}}$. By $(2.16), D_{\mathbf{c}^{(M)}} \rightarrow D_{\mathbf{c}}$ and $D_{\mathbf{b}^{(M)}} \rightarrow D_{\mathbf{b}}$ in $\mathcal{B}_{2}\left(\ell^{2}\right)$-norm as $M \rightarrow \infty$. Using e.g. [GGK, Lem. IV.7.2], we conclude that $\mathcal{T}^{(M)} \rightarrow \mathcal{T}$ in $\mathcal{B}_{1}\left(\ell^{2}\right)$-norm and hence $\operatorname{det}\left(I-\mathcal{T}^{(M)}\right) \rightarrow \operatorname{det}(I-\mathcal{T})$ as $M \rightarrow \infty$, see e.g. [GGK, (IV.5.14)]. Let $\mathbf{d}_{+}=\left(A_{j}^{-1} B_{j} C_{j}\right)_{j \geq 0}$ and $\mathbf{d}_{+}^{(M)}=\left(\chi_{M}(j) A_{j}^{-1} B_{j} C_{j}\right)_{j \geq 0}$. Then the product in the right-hand side of (4.22) is equal to $\operatorname{det}\left(I+D_{\mathbf{d}_{+}}\right)=\lim _{M \rightarrow \infty} \operatorname{det}\left(I+D_{\mathbf{d}_{+}^{(M)}}\right)$ because $D_{\mathbf{d}_{+}^{(M)}} \rightarrow D_{\mathbf{d}_{+}}$in $\mathcal{B}_{1}\left(\ell^{2}\left(\mathbb{Z}_{+} ; \mathbb{C}^{\mathrm{d}}\right)\right)$-norm, as above. Thus, to prove the claim it remains to show that

$$
\mathcal{E}=\lim _{M \rightarrow \infty} \mathcal{E}^{(M)}
$$

where $\mathcal{E}^{(M)}$ is defined as in (4.18) using the solutions $\mathbf{Y}^{(i, M)}$ of equations (4.10) - (4.11) with $B_{j}$ and $C_{j}$ replaced by $B_{j}^{(M)}=\chi_{M}(j) B_{j}$ and $C_{j}^{(M)}=\chi_{M}(j) C_{j}$. Note that $q^{(M)} \leq$ $q$, cf. (4.15). Thus, $N$ in Lemma 4.2 could be fixed independent of $M$. Note that for $i=1, \ldots, n$ (and similarly for $\left.i=n+1, \ldots, \mathrm{d}_{0}\right)$ we have $\mathbf{Y}^{(i)}=(I-T)^{-1}\left(U_{j} Q_{i}\right)_{j>N}$ and $\mathbf{Y}^{(i, M)}=\left(I-T^{(M)}\right)^{-1}\left(U_{j} Q_{i}\right)_{j>N}$ for the operators $T$ and $T^{(M)}$ defined by the right-hand side of (4.10), and that $\|T\|<q<1$ and $\left\|T^{(M)}\right\|<q$ uniformly for $M \in \mathbb{N}$, for the operator norm in $l_{+, \beta}^{\infty}$, cf. (4.16). Also, $U_{j}^{\times, M} \rightarrow U_{j}^{\times}$as $M \rightarrow \infty$ uniformly for $|j| \leq N$, cf. Remark 4.1. Thus, (4.23) is proved as soon as we show that $\left\|T-T^{(M)}\right\| \rightarrow 0$ as $M \rightarrow \infty$. But $T-T^{(M)}$ is again given by the same expression as $T$, see (4.10), except the product $B_{k} C_{k}$ should be replaced by $B_{k} C_{k}-B_{k}^{(M)} C_{k}^{(M)}=\left(1-\chi_{M}(k)\right) B_{k} C_{k}$. We can now use (4.16) to estimate for $i=1, \ldots, n$ the operator norm of $T-T^{(M)}$ in $l_{+, \beta}^{\infty}$ : 


$$
\begin{aligned}
\left\|T-T^{(M)}\right\| & \leq \max \left\{c_{\alpha}, c_{\alpha}^{\prime}\right\} \sum_{k=N}^{\infty}\left(1-\chi_{M}(k)\right) e^{\left(\varkappa_{i}^{+}-\varkappa_{i}^{\prime+}+\delta\right) k}\left\|B_{k} C_{k}\right\| \\
& =\max \left\{c_{\alpha}, c_{\alpha}^{\prime}\right\} \sum_{k=M+1}^{\infty} e^{\left(\varkappa_{i}^{+}-\varkappa_{i}^{\prime+}+\delta\right) k}\left\|B_{k} C_{k}\right\| \rightarrow 0 \text { as } M \rightarrow \infty,
\end{aligned}
$$

using (4.8). A similar argument works for $i=n+1, \ldots, \mathrm{d}_{0}$. Thus, (4.23) holds, and the claim above is verified.

From now on we therefore assume that $\left(B_{j}\right)_{j \in \mathbb{Z}}$ and $\left(C_{j}\right)_{j \in \mathbb{Z}}$ are finitely supported. Note that then assumption (3.3) holds, and thus (3.36) holds. So, to establish (4.22) we need to prove that $\mathcal{E}=\operatorname{det} \mathcal{U}_{0}^{\times}$, where $\mathcal{U}_{0}^{\times}=V_{0}$ is given by (3.35) in terms of the solutions $\mathbf{X}^{ \pm}$of difference equations (3.29) - (3.30).

Consider the following matrix difference equations:

$$
\begin{aligned}
& Z_{j}^{+}-(I-P)=-\sum_{k=j}^{\infty} U_{k+1}^{-1} B_{k} C_{k} U_{k} Z_{k}^{+}, \\
& Z_{j}^{-}-P=\sum_{k=-\infty}^{j-1} U_{k+1}^{-1} B_{k} C_{k} U_{k} Z_{k}^{-},
\end{aligned}
$$

and introduce on $\ell^{\infty}\left(\mathbb{Z} ; \mathbb{C}^{\mathrm{d} \times \mathrm{d}}\right)$ operators $\widetilde{H}_{+}$and $\widetilde{H}_{-}$corresponding to the right-hand sides of $(4.25)-(4.26)$ so that these equations become $\left(I-\widetilde{H}_{+}\right) \mathbf{Z}^{+}=(I-P)_{j \in \mathbb{Z}}$ and $\left(I-\widetilde{H}_{-}\right) \mathbf{Z}^{-}=(P)_{j \in \mathbb{Z}}$. We claim that the operators $I-\widetilde{H}_{ \pm}$are invertible on $\ell^{\infty}\left(\mathbb{Z} ; \mathbb{C}^{\mathrm{d} \times \mathrm{d}}\right)$. Indeed, using notation $(2.5)-(2.6)$ we have $\widetilde{H}_{+}=-D_{\mathbf{d}}-V_{\mathbf{a}, \mathbf{b}}^{+}$and $\widetilde{H}_{-}=$ $V_{\mathbf{a}, \mathbf{b}}^{-}$with $\mathbf{a}=\left(U_{j+1}^{-1} B_{j}\right)_{j \in \mathbb{Z}} \in \ell^{1}, \mathbf{b}=\left(C_{j} U_{j}\right)_{j \in \mathbb{Z}} \in \ell^{1}$, and $\mathbf{d}=\left(U_{j+1}^{-1} B_{j} C_{j} U_{j}\right)_{j \in \mathbb{Z}} \in \ell^{1}$. The operator $I+D_{\mathbf{d}}$ is invertible in $\ell^{\infty}\left(\mathbb{Z} ; \mathbb{C}^{\mathrm{d} \times \mathrm{d}}\right)$ since the operator $I+D_{\mathbf{d}^{\prime}}, \mathbf{d}^{\prime}=$ $\left(B_{j} C_{j} U_{j} U_{j+1}^{-1}\right)_{j \in \mathbb{Z}}$ in invertible in $\ell^{\infty}\left(\mathbb{Z} ; \mathbb{C}^{\mathrm{d} \times \mathrm{d}}\right)$ by assumptions (3.1) and (3.4) and the identities $I+B_{j} C_{j} U_{j} U_{j+1}^{-1}=A_{j}^{\times} A_{j}^{-1}, j \in \mathbb{Z}$ (recall that $A_{j}^{\times}=A_{j}$ for sufficiently large $|j|$ because $\left(B_{j}\right)_{j \in \mathbb{Z}}$ and $\left(C_{j}\right)_{j \in \mathbb{Z}}$ are finitely supported). Moreover, $\left(I+D_{\mathbf{d}}\right)^{-1}=D_{\mathbf{d}^{\prime \prime}}$ with some $\mathbf{d}^{\prime \prime}=\left(d_{j}^{\prime \prime}\right)_{j \in \mathbb{Z}} \in \ell^{\infty}\left(\mathbb{Z} ; \mathbb{C}^{\mathrm{d} \times \mathrm{d}}\right)$. Since

$$
I-\widetilde{H}_{+}=\left(I+D_{\mathbf{d}}\right)\left(I+\left(I+D_{\mathbf{d}}\right)^{-1} V_{\mathbf{a}, \mathbf{b}}^{+}\right)=\left(I+D_{\mathbf{d}}\right)\left(I+V_{\mathbf{a}^{\prime}, \mathbf{b}}^{+}\right)
$$

with $\mathbf{a}^{\prime}=\left(d_{j}^{\prime \prime} U_{j+1}^{-1} B_{j}\right)_{j \in \mathbb{Z}} \in \ell^{1}$, the operators $I-\widetilde{H}_{+}$and $I-\widetilde{H}_{-}$are invertible by (2.12), as claimed. Therefore, equations (4.25) - (4.26) have a unique pair of solutions $\mathbf{Z}^{ \pm} \in \ell^{\infty}\left(\mathbb{Z} ; \mathbb{C}^{\mathrm{d} \times \mathrm{d}}\right)$ given by

$$
\mathbf{Z}^{+}=\left(I-\widetilde{H}_{+}\right)^{-1}(I-P)_{j \in \mathbb{Z}} \text { and } \mathbf{Z}_{-}=\left(I-\widetilde{H}_{-}\right)^{-1}(P)_{j \in \mathbb{Z}} .
$$

In particular $Z_{j}^{+}(I-P)=Z_{j}^{+}$and $Z_{j}^{-} P=Z_{j}^{-}, j \in \mathbb{Z}$. Also, using (4.25) - (4.26), it is easy to see that $\left(U_{j} Z_{j}^{ \pm}\right)_{j \in \mathbb{Z}}$ are solutions of the equation $x_{j+1}=A_{j}^{\times} x_{j}, j \in \mathbb{Z}$. Thus, if $\left(U_{j}^{\times}\right)_{j \in \mathbb{Z}}$ is the fundamental matrix solution of the equation $x_{j+1}=A_{j}^{\times} x_{j}$, then the following relations hold:

$$
Z_{0}^{+}=\left(U_{N+1}^{\times}\right)^{-1}\left(U_{N+1} Z_{N+1}^{+}\right) \text {and } Z_{0}^{-}=\left(U_{-N}^{\times}\right)^{-1}\left(U_{-N} Z_{-N}^{-}\right)
$$

(recall that $U_{0}=U_{0}^{\times}=I$, and $N$ here is chosen as in Lemma 4.2). On the other hand, multiplying (4.25) - (4.26) by $C_{j} U_{j}$ from the left, letting $X_{j}^{ \pm}=C_{j} U_{j} Z_{j}^{ \pm}$and recalling that $\left(C_{j} U_{j}\right)_{j \in \mathbb{Z}}$ is finitely supported, we conclude that $\mathbf{X}^{ \pm}=\left(X_{j}\right)_{j \in \mathbb{Z}} \in \ell^{2}\left(\mathbb{Z} ; \mathbb{C}^{\mathrm{d} \times \mathrm{d}}\right)$ is the 
unique pair of solutions of equations (3.29) - (3.30). Using the direct sum decomposition $\mathbb{C}^{\mathrm{d}}=\operatorname{Im}(I-P) \oplus \operatorname{Im} P$, and writing $Z_{j}^{+}+Z_{j}^{-}, j \in \mathbb{Z}$, as a $(2 \times 2)$-block matrix, we observe that $Z_{j}^{+}+Z_{j}^{-}=V_{j}$, where $V_{j}$ is given in (3.35) in terms of $\mathbf{X}^{ \pm}$. So, to complete the proof of the theorem we need to show that $\mathcal{E}=\operatorname{det}\left[Z_{0}^{+}+Z_{0}^{-}\right]$.

Since $\left(U_{j+1}^{-1} B_{j}\right)_{j \in \mathbb{Z}}$ and $\left(C_{j} U_{j}\right)_{j \in \mathbb{Z}}$ are finitely supported, equations (4.10) - (4.11) can be equivalently rewritten as follows:

$$
\begin{aligned}
Y_{j}^{(i)}= & U_{j} Q_{i}-\sum_{k=j}^{\infty} U_{j} U_{k+1}^{-1} B_{k} C_{k} Y_{k}^{(i)} \\
& +\sum_{k=N}^{\infty} U_{j}\left(I-\left(Q_{i}+\cdots+Q_{\mathrm{d}_{0}}\right)\right) U_{k+1}^{-1} B_{k} C_{k} Y_{k}^{(i)}, \quad j>N, \\
Y_{j}^{(i)}= & U_{j} Q_{i}+\sum_{k=-\infty}^{j-1} U_{j} U_{k+1}^{-1} B_{k} C_{k} Y_{k}^{(i)} \\
& -\sum_{k=-\infty}^{-N} U_{j}\left(I-\left(Q_{1}+\cdots+Q_{i}\right)\right) U_{k+1}^{-1} B_{k} C_{k} Y_{k}^{(i)}, \quad j \leq-N,
\end{aligned}
$$

Recall that $Y_{j}^{(i)} Q_{i}=Y_{j}^{(i)}$ by Lemma 4.2 , and that $I-P=Q_{1}+\cdots+Q_{n}, P=Q_{n+1}+$ $\cdots+Q_{\mathrm{d}_{0}}$, so that $(I-P) Q_{i}=Q_{i}$ for $i=1, \ldots, n$ and $P Q_{i}=Q_{i}$ for $i=n+1, \ldots, \mathrm{d}_{0}$. Using notation $Y_{j}^{+}=\sum_{i=1}^{n} Y_{j}^{(i)}$ and $Y_{j}^{-}=\sum_{i=n+1}^{\mathrm{d}_{0}} Y_{j}^{(i)}$, we derive from (4.29) - (4.30):

$$
\begin{gathered}
Y_{j}^{+}=U_{j}(I-P)-\sum_{k=j}^{\infty} U_{j} U_{k+1}^{-1} B_{k} C_{k} Y_{k}^{+}+U_{j} T_{+}, \quad j>N, \\
Y_{j}^{-}=U_{j} P+\sum_{k=-\infty}^{j-1} U_{j} U_{k+1}^{-1} B_{k} C_{k} Y_{k}^{-}+U_{j} T_{-}, \quad j \leq-N,
\end{gathered}
$$

where we have denoted:

$$
\begin{aligned}
& T_{+}=\sum_{i=1}^{n}\left(I-\left(Q_{i}+\cdots Q_{\mathrm{d}_{0}}\right)\right) \eta_{i} Q_{i}, \quad \eta_{i}=\sum_{k=N}^{\infty} U_{k+1}^{-1} B_{k} C_{k} Y_{k}^{(i)}, \\
& T_{-}=\sum_{i=n+1}^{d_{0}}\left(I-\left(Q_{1}+\cdots Q_{i}\right)\right) \eta_{i} Q_{i}, \quad \eta_{i}=\sum_{k=-\infty}^{-N} U_{k+1}^{-1} B_{k} C_{k} Y_{k}^{(i)} .
\end{aligned}
$$

We remark that, in the direct sum decomposition $\mathbb{C}^{\mathrm{d}}=\operatorname{Im}(I-P) \oplus \operatorname{Im} P$,

$$
T_{+}=(I-P) T_{+}(I-P), \quad T_{-}=P T_{-} P, \quad \text { and } \quad T_{+}+T_{-}=T_{+} \oplus T_{-} .
$$

Using the operators $\widetilde{H}_{ \pm}$, we can also rewrite $(4.31)$ - (4.32) as follows:

$$
\begin{aligned}
\left(I-\widetilde{H}_{+}\right)\left(U_{j}^{-1} Y_{j}^{+}\right)_{j>N} & =\left((I-P)\left(I-P+T_{+}\right)\right)_{j>N}, \\
\left(I-\widetilde{H}_{-}\right)\left(U_{j}^{-1} Y_{j}^{-}\right)_{j \leq-N} & =\left(P\left(P+T_{-}\right)\right)_{j \leq-N} .
\end{aligned}
$$

Comparison with (4.27) yields:

$$
Y_{j}^{+}=U_{j} Z_{j}^{+}\left(I-P+T_{+}\right), j>N, \quad \text { and } \quad Y_{j}^{-}=U_{j} Z_{j}^{-}\left(P+T_{-}\right), j \leq-N .
$$


Formulas (4.12) and (4.28) with the fundamental matrix solution $\left(U_{j}^{\times}\right)_{j \in \mathbb{Z}}$ of $x_{j+1}=$ $A_{j}^{\times} x_{j}$ then imply:

$$
\begin{aligned}
Y_{0}^{+}+Y_{0}^{-} & =\left(U_{N+1}^{\times}\right)^{-1} Y_{N+1}^{+}+\left(U_{-N}^{\times}\right)^{-1} Y_{-N}^{-} \\
& =\left(U_{N+1}^{\times}\right)^{-1}\left(U_{N+1} Z_{N+1}^{+}\left(I-P+T_{+}\right)\right)+\left(U_{-N}^{\times}\right)^{-1}\left(U_{-N} Z_{-N}^{-}\left(P+T_{-}\right)\right) \\
& =Z_{0}^{+}\left(I-P+T_{+}\right)+Z_{0}^{-}\left(P+T_{-}\right)=\left(Z_{0}^{+}+Z_{0}^{-}\right)\left(I+T_{+}+T_{-}\right) .
\end{aligned}
$$

In the last equality we also used the fact that $Z_{0}^{+}=Z_{0}^{+}(I-P)$ and $Z_{0}^{-}=Z_{0}^{-} P$, and assertions (4.35). Since $\mathcal{E}=\operatorname{det}\left[Y_{0}^{+}+Y_{0}^{-}\right]$by (4.18), to finish the proof of the identity $\mathcal{E}=\operatorname{det}\left[Z_{0}^{+}+Z_{0}^{-}\right]$(and thus the proof of the theorem), it suffices to prove that

$$
\operatorname{det}\left[I+T_{+}+T_{-}\right]=1 .
$$

For this, cf. (4.35), we note that the operators $T_{+}=(I-P) T_{+}(I-P)$ on $\operatorname{Im}(I-P)$ and, resp. $T_{-}=P T_{-} P$ on $\operatorname{Im} P$ are represented by lower-, resp. upper-triangular matrices with zero main diagonals in the direct sum decomposition $\operatorname{Im}(I-P)=\operatorname{Im} Q_{1} \oplus \cdots \oplus \operatorname{Im} Q_{n}$, resp. $\operatorname{Im} P=\operatorname{Im} Q_{n+1} \oplus \cdots \oplus \operatorname{Im} Q_{\mathrm{d}_{0}}$ (cf. (4.33) - (4.34)). Thus, the proof of (4.36) and the theorem is completed.

5. Constant coefficients. In this section we specialize to the case where $A_{j} \equiv A, j \in \mathbb{Z}$, with a given matrix $A \in \mathbb{C}^{\mathrm{d} \times \mathrm{d}}$ satisfying the assumptions

$$
0 \notin \sigma(A) \text { and } \sigma(A) \cap\{z \in \mathbb{C}:|z|=1\}=\emptyset,
$$

cf. assumptions (3.1) - (3.2). We will continue to assume that $A_{j}^{\times}=A+B_{j} C_{j}$ is invertible for each $j \in \mathbb{Z}$, cf. (3.4). Note that $U_{j}=A^{j}, j \in \mathbb{Z}$. We will show that in this constant coefficients case assumptions (4.8) - (4.9) of the exponential decay of the perturbation could be replaced with a weaker assumption of a polynomial decay. Specifically, let $m$ denote the maximal size of Jordan blocks in the Jordan canonical form of the $(\mathrm{d} \times \mathrm{d})$ matrix $A$. (Then $m=0$ provided all eigenvalues of $A$ are semi-simple, that is, when all Jordan blocks are diagonal.) The following assumption will replace (4.8) - (4.9):

$$
\sum_{k=-\infty}^{\infty}|k|^{2 m}\left\|B_{k} C_{k}\right\|<\infty .
$$

Let us decompose $\sigma(A)=\cup_{i=1}^{\mathrm{d}_{0}} \sigma_{i}$ where $\sigma_{i}$ 's belong to concentric circles whose radii are denoted by $e^{\varkappa_{i}}$ so that $\sigma_{i}=\left\{\lambda \in \sigma(A):|\lambda|=e^{\varkappa_{i}}\right\}$, and enumerate the numbers $\varkappa_{i}$ so that

$$
\varkappa_{1}<\cdots<\varkappa_{n}<0<\varkappa_{n+1}<\varkappa_{\mathrm{d}_{0}}
$$

for some $n \in\left\{1, \ldots, \mathrm{d}_{0}\right\}$. Let $Q_{i}$ denote the spectral projection for $A$ such that $\sigma\left(\left.A\right|_{\operatorname{Im} Q_{i}}\right)$ $=\sigma_{i}$. Note that $\varkappa_{i}=\varkappa_{g}\left(Q_{i} ; \mathbb{Z}\right)=\varkappa_{g}^{\prime}\left(Q_{i} ; \mathbb{Z}\right)=\varkappa_{i}^{\prime \pm}=\varkappa_{i}^{ \pm}$for the Bohl exponents, cf. (4.6). Passing to appropriate coordinates, we will assume that $A$ is in the Jordan normal form. Thus, each $\left.A\right|_{\operatorname{Im} Q_{i}}$ is a direct sum of (maybe, several) Jordan blocks $\lambda I+E$ with $|\lambda|=e^{\varkappa_{i}}$. By Lemma 2.3,

$$
\left\|\left.A^{j}\right|_{\operatorname{Im} Q_{i}}\right\| \leq c|j|^{m} e^{j \varkappa_{i}}, \quad i=1, \ldots, \mathrm{d}_{0}, \quad j \in \mathbb{Z} .
$$


Equations (4.10) - (4.11) now become:

$$
\begin{aligned}
& \mathbf{Y}^{(i)}-\left(A^{j} Q_{i}\right)_{j>N}=T_{i} \mathbf{Y}^{(i)}, \quad i=1, \ldots, n, \\
& \mathbf{Y}^{(i)}-\left(A^{j} Q_{i}\right)_{j \leq-N}=T_{i} \mathbf{Y}^{(i)}, \quad i=n+1, \ldots, \mathrm{d}_{0},
\end{aligned}
$$

where

$$
\begin{aligned}
\left(T_{i} \mathbf{u}\right)_{j}= & -\sum_{k=j}^{\infty} A^{j-k-1}\left(Q_{i}+\cdots+Q_{\mathrm{d}_{0}}\right) B_{k} C_{k} u_{k} \\
& +\sum_{k=N}^{j-1} A^{j-k-1}\left(I-\left(Q_{i}+\cdots+Q_{\mathrm{d}_{0}}\right)\right) B_{k} C_{k} u_{k}, \quad \mathbf{u}=\left(u_{j}\right)_{j>N}, i=1, \ldots, n, \\
\left(T_{i} \mathbf{u}\right)_{j}= & \sum_{k=-\infty}^{j-1} A^{j-k-1}\left(Q_{1}+\cdots+Q_{i}\right) B_{k} C_{k} u_{k} \\
(5.7) \quad & -\sum_{k=j}^{-N} A^{j-k-1}\left(I-\left(Q_{1}+\cdots+Q_{i}\right)\right) B_{k} C_{k} u_{k}, \quad \mathbf{u}=\left(u_{j}\right)_{j \leq-N}, i=n+1, \ldots, \mathrm{d}_{0} .
\end{aligned}
$$

LEMMA 5.1. Assume (5.1) - (5.2). Then for a sufficiently large $N$ there exist solutions $\mathbf{Y}^{(i)}=\left(Y_{j}^{(i)}\right)_{j>N}, i=1, \ldots, n$, resp. $\mathbf{Y}^{(i)}=\left(Y_{j}^{(i)}\right)_{j \leq-N}, i=n+1, \ldots, \mathrm{d}_{0}$, of (5.5), resp. (5.6) such that $Y_{j}^{(i)}=Y_{j}^{(i)} Q_{i}$ and the following assertions hold:

(a)

$$
\begin{aligned}
& \sup _{j>N}|j|^{-m} e^{-j \varkappa_{i}}\left\|Y_{j}^{(i)}\right\|<\infty, \quad i=1, \ldots, n, \\
& \sup _{j \leq-N}|j|^{-m} e^{-j \varkappa_{i}}\left\|Y_{j}^{(i)}\right\|<\infty, \quad i=n+1, \ldots, \mathrm{d}_{0},
\end{aligned}
$$

$$
e^{-j \varkappa_{i}}\left\|Y_{j}^{(i)}-A^{j} Q_{i}\right\| \rightarrow 0
$$

as $j \rightarrow \infty$ for $i=1, \ldots, n$ and as $j \rightarrow-\infty$ for $i=n+1, \ldots, \mathrm{d}_{0}$.

Proof. We prove the lemma for $i=n+1, \ldots, \mathrm{d}_{0}$; the proof for $i=1, \ldots, n$ is similar. Using (5.3) - (5.4), we infer:

$$
\begin{aligned}
& \left\|A^{j-k-1}\left(Q_{1}+\cdots+Q_{i}\right)\right\| \leq c|j-k|^{m} e^{(j-k) \varkappa_{i}}, \quad 0 \geq j \geq k, \\
& \begin{aligned}
\left\|A^{j-k-1}\left(I-\left(Q_{1}+\cdots+Q_{i}\right)\right)\right\| & \leq c|j-k|^{m} e^{(j-k) \varkappa_{i+1}} \\
& \leq c^{\prime}|j-k|^{m} e^{(j-k) \varkappa_{i}}, \quad 0 \geq k \geq j .
\end{aligned}
\end{aligned}
$$

Introduce the Banach space $\ell_{-}^{\infty}=\left\{\mathbf{u}=\left(u_{j}\right)_{j \leq-N}:\|u\|_{\ell_{-}^{\infty}}:=\sup _{j \leq-N}|j|^{-m} e^{-j \varkappa_{i}}\left\|u_{j}\right\|\right.$ $<\infty\}$. Denote $q_{N}=\sum_{k=-\infty}^{-N}|k|^{2 m}\left\|B_{k} C_{k}\right\|$ and remark that $q_{N} \rightarrow 0$ as $N \rightarrow \infty$ by (5.2). Using (5.9) - (5.10) and the fact that $\sup _{k \geq 0}|k|^{m} e^{-k\left(\varkappa_{i+1}-\varkappa_{i}\right)}<\infty$, we have for $j \leq-N$ and the operator $T=T_{i}(N)$ defined in (5.7):

$$
|j|^{-m} e^{-j \varkappa_{i}}\left\|(T \mathbf{u})_{j}\right\| \leq c\|\mathbf{u}\|_{\ell_{-}^{\infty}}\left(\sum_{k=-\infty}^{j-1}\left|j^{-1}(j-k) k\right|^{m}\left\|B_{k} C_{k}\right\|\right.
$$

$$
\left.+\sum_{k=j}^{-N}\left(|j-k|^{m} e^{(j-k)\left(\varkappa_{i+1}-\varkappa_{i}\right)}\right)\left|j^{-1} k\right|^{m}\left\|B_{k} C_{k}\right\|\right) \leq c^{\prime} q_{N}\|\mathbf{u}\|_{\ell_{-}^{\infty}}
$$


Note that $|k| \geq|j| \geq N \geq 1$ in the first sum and $|j| \geq|k| \geq N \geq 1$ in the second sum. Thus, $\|T \mathbf{u}\|_{\ell_{-}^{\infty}} \leq c q_{N}\|\mathbf{u}\|_{\ell_{-}^{\infty}}$, and $T=T_{i}(N)$ is a contraction on $\ell_{-}^{\infty}$ for a sufficiently large $N$. Since $\left(A^{j} Q_{i}\right)_{j \leq-N} \in \ell_{-}^{\infty}$ by (5.4), assertion (5.8) follows. To show (b) in the lemma, we follow the proof of the celebrated Levinson Theorem in [E, p.14]. Indeed, for $j<-M<-N$ and $\mathbf{u}=\left(Y_{j}^{(i)}\right)_{j \leq-N} \in \ell_{-}^{\infty}$, similarly to (5.11), we infer:

$$
\begin{aligned}
& e^{-j \varkappa_{i}}\left\|(T \mathbf{u})_{j}\right\| \leq\left\|e^{-j \varkappa_{i}}\left(T_{i}(M) \mathbf{u}\right)_{j}\right\| \\
& \quad+\sum_{k=-M+1}^{-N}\left\|A^{j-k-1}\left(I-\left(Q_{1}+\cdots+Q_{i}\right)\right) B_{k} C_{k} u_{k}\right\| \\
& \leq c\|\mathbf{u}\|_{\ell_{-}^{\infty}}\left(\sum_{k=-\infty}^{j-1}|(j-k) k|^{m}\left\|B_{k} C_{k}\right\|+\sum_{k=j}^{-M}|j-k|^{m} e^{(j-k)\left(\varkappa_{i+1}-\varkappa_{i}\right)}|k|^{m}\left\|B_{k} C_{k}\right\|\right. \\
& \left.\quad+\sum_{k=-M+1}^{-N}\left(|j-k|^{m} e^{\frac{1}{2}(j-k)\left(\varkappa_{i+1}-\varkappa_{i}\right)}\right) e^{\frac{1}{2}(j-k)\left(\varkappa_{i+1}-\varkappa_{i}\right)}|k|^{m}\left\|B_{k} C_{k}\right\|\right) \\
& \leq c^{\prime}\|\mathbf{u}\|_{\ell_{-}^{\infty}}\left(q_{M}+c(M, N) e^{\frac{1}{2} j\left(\varkappa_{i+1}-\varkappa_{i}\right)}\right) .
\end{aligned}
$$

Choosing first a sufficiently large $M$, and then letting $j \rightarrow-\infty$, we have $(b)$ in the lemma.

As soon as the existence of the solutions of (5.5) - (5.6) is established, we can use formulas (4.12) to obtain the matrix solutions $\mathbf{Y}^{+}=\mathbf{Y}^{(1)}+\cdots+\mathbf{Y}^{(n)}$ on $\mathbb{Z}_{+}$and $\mathbf{Y}^{-}=\mathbf{Y}^{(n+1)}+\cdots+\mathbf{Y}^{\left(\mathrm{d}_{0}\right)}$ on $\mathbb{Z}_{-}$of the perturbed equation $x_{j+1}=\left(A+B_{j} C_{j}\right) x_{j}$ and then define the Evans determinant, $\mathcal{E}$, as indicated in (4.18). Note that the equality $\mathcal{E}=\mathcal{D}$ for the "standard" Evans determinant $\mathcal{D}$ also holds as in Proposition 4.5 since $\mathbf{Y}^{ \pm}$ enjoy the properties listed in Lemma 5.1. The proof of Theorem 4.6 remains unchanged with a natural replacement of the exponential term in (4.24) by $k^{2 m}$. Thus, we have the following result.

Proposition 5.2. Formula (4.22) holds provided $A=A_{j}, j \in \mathbb{Z}$, assumption (3.4) is satisfied, and assumptions (3.1) - (3.2) and (4.8) - (4.9) in Theorem 4.6 are replaced, respectively, by (5.1) and (5.2).

Next, as an illustration, we will consider a particularly important class of second order difference equations, the discrete Schrödinger equation, and show how to specialize our results for the corresponding $(2 \times 2)$ first order system. Given a real-valued potential $\mathbf{v}=$ $\left(v_{j}\right)_{j \in \mathbb{Z}} \in \ell^{1}(\mathbb{Z} ; \mathbb{R})$, consider on $\ell^{2}(\mathbb{Z} ; \mathbb{C})$ a bounded self-adjoint operator, $L$, defined by $(L \mathbf{y})_{j}=y_{j+1}+y_{j-1}+v_{j} y_{j}, \mathbf{y} \in \ell^{2}(\mathbb{Z} ; \mathbb{C})$. If $(\mathcal{S} \mathbf{y})_{j}=y_{j-1}$ denotes the shift operator, and $z \in \mathbb{R}$, then the following identity, cf. [LS, Exmp. 1.6], holds on $\left(\ell^{2}(\mathbb{Z} ; \mathbb{C})\right)^{2}=\ell^{2}\left(\mathbb{Z} ; \mathbb{C}^{2}\right)$ for the operator $L=\mathcal{S}+\mathcal{S}^{-1}+D_{\mathbf{v}}$, cf. (2.6):

$$
\left[\begin{array}{ll}
0 & I \\
I & \mathcal{S}
\end{array}\right]\left[\begin{array}{cc}
L-z I & 0 \\
0 & I
\end{array}\right]\left[\begin{array}{cc}
0 & \mathcal{S} \\
I & -\mathcal{S}
\end{array}\right]=\left[\begin{array}{ll}
I & 0 \\
0 & I
\end{array}\right]+\left[\begin{array}{cc}
0 & -I \\
I & D_{\mathbf{v}}-z I
\end{array}\right]\left[\begin{array}{ll}
\mathcal{S} & 0 \\
0 & \mathcal{S}
\end{array}\right] .
$$

Writing $v_{j}=e^{\mathrm{i} \arg v_{j}}\left|v_{j}\right|$, and introducing $(2 \times 2)$ matrices

$$
A=\left[\begin{array}{cc}
0 & 1 \\
-1 & z
\end{array}\right], \quad B=\left[\begin{array}{cc}
0 & 0 \\
0 & -e^{\mathrm{i} \arg v_{j}}\left|v_{j}\right|^{\frac{1}{2}}
\end{array}\right], \quad C=\left[\begin{array}{cc}
0 & 0 \\
0 & \left|v_{j}\right|^{\frac{1}{2}}
\end{array}\right],
$$


and $A_{j}^{\times}=A+B_{j} C_{j}$, we observe from (5.12) that the operator $L-z I$ has a bounded inverse on $\ell^{2}(\mathbb{Z} ; \mathbb{C})$ if and only if the operator $I-D_{\mathbf{A} \times} \mathcal{S}, \mathbf{A}^{\times}=\left(A_{j}^{\times}\right)_{j \in \mathbb{Z}}$, has a bounded inverse on $\ell^{2}\left(\mathbb{Z} ; \mathbb{C}^{2}\right)$ since the $(2 \times 2)$ operator matrices containing $\mathcal{S}$ in the left-hand side of $(5.12)$ are invertible operators on $\ell^{2}\left(\mathbb{Z} ; \mathbb{C}^{2}\right)$, and the right-hand side of $(5.12)$ is $I-D_{\mathbf{A} \times} \mathcal{S}$. Recalling the operators $G_{\mathbf{A}}$ and $G_{\mathbf{A} \times}$, see the Introduction, corresponding to the difference equations $x_{j+1}=A x_{j}$ and $x_{j+1}=A_{j}^{\times} x_{j}, x_{j} \in \mathbb{C}^{2}, j \in \mathbb{Z}$, by the formulas $\left(G_{\mathbf{A}} \mathbf{x}\right)_{j}=x_{j+1}-A x_{j}$ and $\left(G_{\mathbf{A} \times \mathbf{x}}\right)_{j}=x_{j+1}-A_{j}^{\times} x_{j}$, observe that $\left(I-D_{\mathbf{A} \times} \mathcal{S}\right) \mathcal{S}^{-1}=G_{\mathbf{A} \times}$. Note that $\operatorname{det} A_{j}^{\times}=1$ and thus assumption (3.4) holds. The eigenvalues of $A=A(z)$ will be denoted by $\lambda=\lambda(z)$ and $\lambda^{-1}$; these are the roots of the equation $\lambda^{2}-z \lambda+1=0$. If $|z| \leq 2$ then $|\lambda|=1$; if $|z|>2$ we choose $\lambda$ so that $|\lambda|<1$ and denote $\Lambda=\lambda^{-1}-\lambda$. Thus, if $|z|>2$ then $A=A(z)$ satisfies assumptions (5.1). Then $G_{\mathbf{A}}$ is invertible on $\ell\left(\mathbb{Z} ; \mathbb{C}^{2}\right)$, and the kernel of the operator $\mathcal{K}=G_{\mathbf{A}}^{-1}$ is given by (1.2) where $U_{j}=A^{j}$ and $P=P(z)$ is the spectral projection for $A$ so that $\sigma\left(\left.A\right|_{\operatorname{Im} P}\right)=\left\{\lambda^{-1}\right\}$, explicitly, $P=\frac{1}{\Lambda}\left[\begin{array}{cc}-\lambda & 1 \\ -1 & \lambda^{-1}\end{array}\right]$. Using the identity $G_{\mathbf{A} \times}=G_{\mathbf{A}}\left(I-G_{\mathbf{A}}^{-1} D_{\mathbf{B}} D_{\mathbf{C}}\right)$ with $\mathbf{B}=\left(B_{j}\right)_{j \in \mathbb{Z}} \in \ell^{2}$ and $\mathbf{C}=\left(C_{j}\right)_{j \in \mathbb{Z}} \in \ell^{2}$, recalling that $\mathcal{T}=\mathcal{T}(z)$ from (1.1) satisfies $\mathcal{T}=D_{\mathbf{C}} G_{\mathbf{A}}^{-1} D_{\mathbf{B}}$, and noting that the operators $I-G_{\mathbf{A}}^{-1} D_{\mathbf{B}} D_{\mathbf{C}}$ and $I-\mathcal{T}$ are invertible at the same time, we have the following corollary of Theorem 4.6.

Proposition 5.3. Assume $\mathbf{v}=\left(v_{j}\right)_{j \in \mathbb{Z}} \in \ell^{1}(\mathbb{Z} ; \mathbb{R})$. If $|z|>2$ then $z \in \sigma(L)$ on $\ell^{2}(\mathbb{Z} ; \mathbb{C})$ if and only if $\mathcal{E}(z)=0$ for the Evans function $\mathcal{E}(z)=\operatorname{det}(I-\mathcal{T}(z))$.

We remark that the Evans determinant $\mathcal{E}(z)$ here is defined, cf. $(4.18)$, as $\mathcal{E}(z)=$ $\operatorname{det}\left[Y_{0}^{+}+Y_{0}^{-}\right]$, where $\mathbf{Y}^{ \pm}=\mathbf{Y}^{ \pm}(z)$ are the $(2 \times 2)$ matrix solutions of the following system:

$$
\begin{aligned}
Y_{j}^{+}-A^{j}(z)(I-P(z)) & =-\sum_{k=j}^{\infty} A^{j-k-1}(z) B_{k} C_{k} Y_{k}^{+}, \quad j \in \mathbb{Z}, \\
Y_{j}^{-}-A^{j}(z) P(z) & =\sum_{k=-\infty}^{j-1} A^{j-k-1}(z) B_{k} C_{k} Y_{k}^{-}, \quad j \in \mathbb{Z} .
\end{aligned}
$$

Recall that the solutions satisfy $Y_{j}^{+}=Y_{j}^{+}(I-P(z)), Y_{j}^{-}=Y_{j}^{-} P(z)$. Also, because (5.14) are Volterra-type equations, passing to $\lambda^{\mp j} Y_{j}^{ \pm}$and using (2.12), one can easily see that $\mathbf{Y}^{ \pm}=\left(Y_{j}^{ \pm}\right)$are indeed defined for all $j \in \mathbb{Z}$.

Next, we will use the special structure of $A, B_{j}$, and $C_{j}$ to simplify system (5.14). Introduce matrices

$$
W=\left[\begin{array}{cc}
1 & 1 \\
\lambda & \lambda^{-1}
\end{array}\right], \quad W^{-1}=\Lambda^{-1}\left[\begin{array}{cc}
\lambda^{-1} & -1 \\
-\lambda & 1
\end{array}\right], \quad V=\left[\begin{array}{cc}
-1 & -1 \\
1 & 1
\end{array}\right], \quad \widetilde{A}=\left[\begin{array}{cc}
\lambda & 0 \\
0 & \lambda^{-1}
\end{array}\right],
$$

and remark that $\widetilde{A}=W^{-1} A W$ and $\Lambda^{-1} v_{j} V \widetilde{A}=W^{-1} B_{j} C_{j} W$ for the matrices $A, B_{j}$, and $C_{j}$ introduced in (5.13). The change of variables $\widetilde{x}_{j}=W^{-1} x_{j}$ transforms the equation $x_{j+1}=A_{j}^{\times} x_{j}$ to the equation $\widetilde{x}_{j+1}=\left(\widetilde{A}+\Lambda^{-1} v_{j} V \widetilde{A}\right) \widetilde{x}_{j}$ with the diagonal unperturbed coefficient $\widetilde{A}$ whose dichotomy projection $\widetilde{P}=W^{-1} P W$ is given by $\widetilde{P}=\left[\begin{array}{ll}0 & 0 \\ 0 & 1\end{array}\right]$. Similarly, passing in equations (5.14) to the new unknowns $\tilde{\mathbf{Y}}^{ \pm}=W^{-1} \mathbf{Y}^{ \pm} W$, we may use the fact that $\mathbf{Y}^{+}=\mathbf{Y}^{+}(I-P)$ and $\mathbf{Y}^{-}=\mathbf{Y}^{-} P$ imply $\widetilde{\mathbf{Y}}^{+}=\widetilde{\mathbf{Y}}^{+}(I-\widetilde{P})$ and $\tilde{\mathbf{Y}}^{-}=\widetilde{\mathbf{Y}}^{-} \widetilde{P}$ to 
conclude that the second column in $\widetilde{\mathbf{Y}}_{j}^{+}$and the first column in $\widetilde{\mathbf{Y}}_{j}^{-}$are equal to zero. Let $u_{j}^{+} \in \mathbb{C}^{2}$, resp. $u_{j}^{-} \in \mathbb{C}^{2}$ denote the first column of $\widetilde{\mathbf{Y}}_{j}^{+}$, resp. the second column of $\tilde{\mathbf{Y}}_{j}^{-}$. Then equations $(5.14)$ are equivalent to the following $(2 \times 1)$ vector equations:

$$
\begin{aligned}
& u_{j}^{+}=\left[\begin{array}{ll}
\lambda^{j} & 0
\end{array}\right]^{\top}-\sum_{k=j}^{\infty} \Lambda^{-1} v_{k} \widetilde{A}^{j-k-1} V \widetilde{A} u_{k}^{+}, \quad j \in \mathbb{Z}, \\
& u_{j}^{-}=\left[\begin{array}{ll}
0 & \lambda^{-j}
\end{array}\right]^{\top}-\sum_{k=-\infty}^{j-1} \Lambda^{-1} v_{k} \widetilde{A}^{j-k-1} V \widetilde{A} u_{k}^{-}, \quad j \in \mathbb{Z} .
\end{aligned}
$$

Introducing $(2 \times 1)$ vectors $x_{j}^{ \pm}=W u_{j}^{ \pm}$given, in components, by $x_{j}^{ \pm}=\left[x_{j}^{ \pm}(1) \quad x_{j}^{ \pm}(2)\right]^{\top}$ and passing in (5.15) to the new unknowns $x_{j}^{ \pm}$, we have the following system of equations equivalent to (5.14):

$$
\begin{aligned}
& x_{j}^{+}=\left[\begin{array}{ll}
\lambda^{j} & \lambda^{j+1}
\end{array}\right]^{\top}-\sum_{k=j}^{\infty} \Lambda^{-1} v_{k}\left[\begin{array}{ll}
\lambda^{-j+k+1}-\lambda^{j-k-1} & \lambda^{-j+k}-\lambda^{j-k}
\end{array}\right]^{\top} x_{k}^{+}(2), \\
& x_{j}^{-}=\left[\begin{array}{ll}
\lambda^{-j} & \lambda^{-j-1}
\end{array}\right]^{\top}+\sum_{k=-\infty}^{j-1} \Lambda^{-1} v_{k}\left[\begin{array}{ll}
\lambda^{-j+k+1}-\lambda^{j-k-1} & \lambda^{-j+k}-\lambda^{j-k}
\end{array}\right]^{\top} x_{k}^{-}(2) .
\end{aligned}
$$

Recall that $\mathbf{y}=\left(y_{j}\right)_{j \in \mathbb{Z}}, y_{j} \in \mathbb{C}$, is a solution of the difference equation $L \mathbf{y}=z \mathbf{y}$ if and only if $\mathbf{x}=\left(x_{j}\right)_{j \in \mathbb{Z}}$ with $x_{j}=\left[\begin{array}{ll}y_{j-1} & y_{j}\end{array}\right]^{\top} \in \mathbb{C}^{2}$ is a solution of the equation $x_{j+1}=A_{j}^{\times} x_{j}$. Moreover, since $\operatorname{det} A_{j}^{\times}=1$, if $\mathbf{y}^{+}$and $\mathbf{y}^{-}$are two solutions of the equation $L \mathbf{y}=z \mathbf{y}$ with the corresponding $\mathbf{x}^{+}$and $\mathbf{x}^{-}$, then the Wronskian

$$
\mathcal{W}\left(\mathbf{y}^{+}, \mathbf{y}^{-}\right):=y_{j-1}^{+} y_{j}^{-}-y_{j}^{+} y_{j-1}^{-}=\operatorname{det}\left[x_{j}^{+} \quad x_{j}^{-}\right]
$$

does not depend on $j \in \mathbb{Z}$.

A direct calculation shows that solutions $\mathbf{x}^{ \pm}=\left(x_{j}^{ \pm}\right)$of (5.16) have the property $x_{j+1}^{ \pm}(1)=x_{j}^{ \pm}(2)$. Also, $\mathbf{x}^{ \pm}$satisfy the equation $x_{j+1}^{ \pm}=A_{j}^{\times} x_{j}^{ \pm}$because $\mathbf{Y}^{ \pm}$in (5.14) satisfy this equation. Thus, letting $y_{j}^{ \pm}=\lambda^{\mp 1} x_{j}^{ \pm}(2)$ and keeping only the second component of the vectors in (5.16), we have obtained the solutions $\mathbf{y}^{ \pm}=\left(y_{j}^{ \pm}\right)$of the second order difference equation $L \mathbf{y}=z \mathbf{y}$ that satisfy the following equations:

$$
\begin{aligned}
& y_{j}^{+}=\lambda^{j}-\sum_{k=j}^{\infty} \Lambda^{-1} v_{k}\left(\lambda^{-j+k}-\lambda^{j-k}\right) y_{k}^{+}, \quad j \in \mathbb{Z}, \\
& y_{j}^{-}=\lambda^{-j}+\sum_{k=-\infty}^{j-1} \Lambda^{-1} v_{k}\left(\lambda^{-j+k}-\lambda^{j-k}\right) y_{k}^{-}, \quad j \in \mathbb{Z} .
\end{aligned}
$$

Introducing $\varkappa<0$ so that $\lambda=e^{\varkappa},(5.18)$ could be rewritten as follows:

$$
\begin{aligned}
& y_{j}^{+}=e^{j \varkappa}-\sum_{k=j}^{\infty} v_{k} \frac{\sinh (j-k) \varkappa}{\sinh \varkappa} y_{k}^{+}, \quad j \in \mathbb{Z}, \\
& y_{j}^{-}=e^{-j \varkappa}+\sum_{k=-\infty}^{j-1} v_{k} \frac{\sinh (j-k) \varkappa}{\sinh \varkappa} y_{k}^{-}, \quad j \in \mathbb{Z} .
\end{aligned}
$$


The solutions $\mathbf{y}^{ \pm}=\left(y_{j}^{ \pm}\right)_{j \in \mathbb{Z}_{ \pm}}$are asymptotic to $\lambda^{ \pm j}=e^{ \pm j \varkappa}$ as $j \rightarrow \pm \infty$; these are the Jost solutions of $L \mathbf{y}=z \mathbf{y}$, cf. [CS, XVII.1.9-10], [GH, Sec. 2], [GM, (4.52)] for the continuous and [C, C1], [FT, Sec. III.2], [GH, (6.6)], [HKS], [T, (10.3)], and [To, (3.3.1)] for the discrete models. Recalling that $\mathbf{y}^{ \pm}=\mathbf{y}^{ \pm}(z)$ and definition (5.17) of the Wronskian of the solutions, we define the Jost function, $\mathcal{J}=\mathcal{J}(z)$, by $\mathcal{J}(z):=\Lambda^{-1} \mathcal{W}\left(\mathbf{y}^{+}(z), \mathbf{y}^{-}(z)\right)$, cf. [T, Sec. 10.2] and [To, (3.3.19)]. Our last claim is that, in fact, the Evans function for the Schrödinger equation is the same as the Jost function.

Proposition 5.4. If $\mathbf{v}=\left(v_{j}\right)_{j \in \mathbb{Z}} \in \ell^{1}(\mathbb{Z} ; \mathbb{R})$ and $|z|>2$ then $\mathcal{E}(z)=\mathcal{J}(z)$.

Proof. Using the choice of transformations converting (5.14) to (5.18), we infer:

$$
\begin{aligned}
\mathcal{E}(z) & =\operatorname{det}\left[Y_{0}^{+}+Y_{0}^{-}\right]=\operatorname{det}\left[W \tilde{Y}_{0}^{+} W^{-1}+W \widetilde{Y}_{0}^{-} W^{-1}\right]=\operatorname{det} W \operatorname{det}\left[\tilde{Y}_{0}^{+}+\tilde{Y}_{0}^{-}\right] \operatorname{det} W^{-1} \\
& =\operatorname{det}\left[u_{0}^{+} \quad u_{0}^{-}\right]=\operatorname{det}\left[W^{-1} x_{0}^{+} \quad W^{-1} x_{0}^{-}\right]=\Lambda^{-1} \operatorname{det}\left[x_{0}^{+} \quad x_{0}^{-}\right] \\
& =\Lambda^{-1} \mathcal{W}\left(\mathbf{y}^{+}, \mathbf{y}^{-}\right)=\mathcal{J}(z),
\end{aligned}
$$

which concludes the proof.

\section{References}

[AGJ] J. Alexander, R. Gardner and C. Jones, A topological invariant arising in the stability analysis of travelling waves, J. Reine Angew. Math. 410 (1990), 167-212.

[BCK] N. Balmforth, R. Craster and P. Kevrekidis, Being stable and discrete, Phys. D 135 (2000), 212-232.

[C] K. Case, Orthogonal polynomials from the viewpoint of scattering theory, J. Math. Phys. 15 (1974), 2166-2174.

[C1] K. Case, On discrete inverse scattering problems. II, J. Math. Phys. 14 (1973), 916920.

[CS] K. Chadan and P. Sabatier, Inverse Problems in Quantum Scattering Theory, 2nd ed., Springer-Verlag, New York, 1989.

[CL] C. Chicone and Y. Latushkin, Evolution Semigroups in Dynamical Systems and Differential Equations, Math. Surv. Monogr. 70, Amer. Math. Soc., Providence, 1999.

[Co] W. A. Coppel, Dichotomies in Stability Theory, Lect. Notes in Math. 629, SpringerVerlag, New York, 1978.

[DK] J. Daleckij and M. Krein, Stability of Differential Equations in Banach Space, Amer. Math. Soc., Providence, RI, 1974.

[E] M. Eastham, The Asymptotic Solution of Linear Differential Systems. Applications of the Levinson Theorem, Oxford Univ. Press, New York, 1989.

[FT] L. Faddeev and L. Takhtajan, Hamiltonian Methods in the Theory of Solitons, Springer-Verlag, Berlin, 1987.

[GH] F. Gesztesy and H. Holden, Trace formulas and conservation laws for nonlinear evolution equations, Rev. Math. Phys. 6 (1994) 51-95.

[GML] F. Gesztesy, Y. Latushkin and K. A. Makarov, Evans functions, Jost functions, and Fredholm determinants, preprint.

[GML1] F. Gesztesy, Y. Latushkin and K. A. Makarov, Evans functions and modified Fredholm determinants, Report No. 36/2004, Oberwolfach Reports 1 (2004), 1946-1948. 
[GM] F. Gesztesy and K. A. Makarov, (Modified) Fredholm determinants for operators with matrix-valued semi-separable integral kernels revisited, Integral Equations Operator Theory 47 (2003), 457-497; erratum: 48 (2004) 425-426; corrected (electronic only) version: 48 (2004) 561-602, DOI 10.1007/s00020-003-1279-z.

[GGK] I. Gohberg, S. Goldberg and N. Krupnik, Traces and Determinants of Linear Operators, Operator Theory Adv. Appl. 116, Birkhäuser, Basel, 2000.

[GKvS] I. Gohberg, M. Kaashoek and F. van Schagen, Noncompact integral operators with semiseparable kernels and their discrete analogues: inversion and Fredholm properties, Integral Equations Operator Theory 7 (1984), 642-703.

[HKS] D. Hinton, M. Klaus and J. Shaw, Half-bound states and Levinson's theorem for discrete systems, SIAM J. Math. Anal. 22 (1991), 754-768.

[JK] C. Jones and T. Kapitula, Stability of fronts, pulses, and wave-trains, preprint, 2003.

[KK] T. Kapitula and P. Kevrekidis, Stability of waves in discrete systems, Nonlinearity 14 (2001), 533-566.

[KS] T. Kapitula and B. Sandstede, Eigenvalues and resonances using the Evans function, Discrete Contin. Dyn. Syst. 10 (2004), 857-869.

[KS1] T. Kapitula and B. Sandstede, Edge bifurcations for near integrable systems via Evans function techniques, SIAM J. Math. Anal. 33 (2002) 1117-1143.

[LS] Y. Latushkin and A. M. Stepin, Weighted shift operators, spectral theory of linear extensions and the multiplicative ergodic theorem, Matem. Sbornik 70 (1991), 143-163.

[SS] R. Sacker and G. Sell, A spectral theory for linear differential systems, J. Differential Equations 27 (1978), 320-358.

[S] B. Sandstede, Stability of travelling waves, in: Handbook of Dynamical Systems, Vol. 2, North-Holland, Amsterdam, 2002, 983-1055.

[Si] B. Simon, Trace Ideals and their Applications, Cambridge Univ. Press, Cambridge, 1979.

[T] G. Teschl, Jacobi Operators and Completely Integrable Nonlinear Lattices, Math. Surv. Monogr. 72, Amer. Math. Soc., Providence, RI, 2000.

[To] M. Toda, Theory of Nonlinear Lattices, 2nd ed., Springer-Verlag, Berlin, 1989. 
